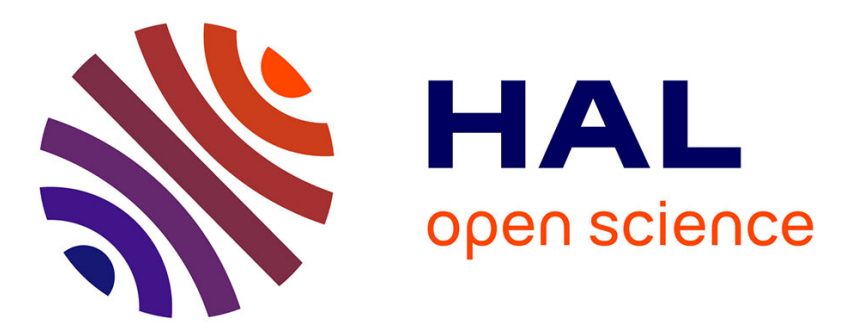

\title{
Uniqueness results for diagonal hyperbolic systems with large and monotone data
}

\author{
Ahmad El Hajj, Régis Monneau
}

\section{To cite this version:}

Ahmad El Hajj, Régis Monneau. Uniqueness results for diagonal hyperbolic systems with large and monotone data. Journal of Hyperbolic Differential Equations, 2013, 10 (3), pp.461-494. 10.1142/S0219891613500161. hal-00534134v2

HAL Id: hal-00534134

https://hal.science/hal-00534134v2

Submitted on 13 Dec 2010

HAL is a multi-disciplinary open access archive for the deposit and dissemination of scientific research documents, whether they are published or not. The documents may come from teaching and research institutions in France or abroad, or from public or private research centers.
L'archive ouverte pluridisciplinaire HAL, est destinée au dépôt et à la diffusion de documents scientifiques de niveau recherche, publiés ou non, émanant des établissements d'enseignement et de recherche français ou étrangers, des laboratoires publics ou privés. 


\title{
Some uniqueness results for diagonal hyperbolic systems with large and monotone data
}

\author{
A. El HajJ ${ }^{1}$, R. Monneau ${ }^{2}$
}

December 13, 2010

\begin{abstract}
In this paper, we study the uniqueness of solutions for diagonal hyperbolic systems in one space dimension. We present two uniqueness results. The first one is a global existence and uniqueness result of a continuous solution for strictly hyperbolic systems. The second one is a global existence and uniqueness result of a Lipschitz solution for hyperbolic systems not necessarily strictly hyperbolic. An application of these two results is shown in the case of one-dimensional isentropic gas dynamics.
\end{abstract}

AMS Classification: 35L45, 35Q35, 35Q72, 74H25.

Key words: Uniqueness results, system of Burgers equations, system of nonlinear transport equations, nonlinear hyperbolic system, isentropic gas dynamics.

\section{Introduction and main results}

\subsection{Setting of the problem}

In this paper we are interested in continuous solutions to hyperbolic systems in dimension one. Our work will focus on solutions $u(t, x)=\left(u^{i}(t, x)\right)_{i=1, \ldots, d}$, where $d \geq 1$ is an integer, of hyperbolic systems which are diagonal, i.e.

$$
\partial_{t} u^{i}+\lambda^{i}(u) \partial_{x} u^{i}=0 \quad \text { on } \quad(0,+\infty) \times \mathbb{R}, \quad \text { for } \quad i=1, \ldots, d,
$$

with the initial data:

$$
u^{i}(0, x)=u_{0}^{i}(x), \quad x \in \mathbb{R}, \quad \text { for } \quad i=1, \ldots, d .
$$

Here we use the notation $\partial_{t}=\frac{\partial}{\partial t}$ and $\partial_{x}=\frac{\partial}{\partial x}$. Such systems are (sometimes) called $(d \times d)$ diagonal hyperbolic systems.

\footnotetext{
${ }^{1}$ Université de Technologie de Compiègne, Département Génie Informatique, 60205 COMPIEGNE Cedex, France

${ }^{2}$ Université Paris-Est, Ecole des Ponts ParisTech, CERMICS, 6 et 8 avenue Blaise Pascal, Cité Descartes Champs-sur-Marne, 77455 Marne-la-Vallée Cedex 2, France
} 
For real numbers $\alpha^{i} \leq \beta^{i}$, let us consider the box

$$
U=\Pi_{i=1}^{d}\left[\alpha^{i}, \beta^{i}\right] .
$$

We consider a given function $\lambda=\left(\lambda^{i}\right)_{i=1, \ldots, d}: U \rightarrow \mathbb{R}^{d}$, which satisfies the following regularity assumption:

$$
(H 1)\left\{\begin{array}{l}
\lambda \in\left[C^{\infty}(U)\right]^{d}, \\
\text { there exists } M_{0}>0 \quad \text { such that for } i=1, \ldots, d, \\
\left|\lambda^{i}(u)\right| \leq M_{0} \text { for all } u \in U, \\
\text { there exists } M_{1}>0 \text { such that for } i=1, \ldots, d, \\
\left|\lambda^{i}(v)-\lambda^{i}(u)\right| \leq M_{1}|v-u| \text { for all } v, u \in U,
\end{array}\right.
$$

where $|w|=\sum_{i=1, \ldots, d}\left|w^{i}\right|$, for $w=\left(w^{1}, \ldots, w^{d}\right)$. Given any Banach space $\left(E,\|\cdot\|_{E}\right)$, in the rest of the paper we consider the norm on $E^{d}$ :

$$
\|w\|_{E^{d}}=\sum_{i=1, \ldots, d}\left\|w^{i}\right\|_{E}, \quad \text { for } \quad w=\left(w^{1}, \ldots, w^{d}\right) \in E^{d} .
$$

Then, we define

$$
\lambda_{, j}^{i}(u)=\frac{\partial \lambda^{i}}{\partial u^{j}}(u), \quad \text { for } \quad i, j=1, \ldots, d,
$$

and we assume that

$$
(H 2) \quad \lambda_{, i}^{i}(u) \geq 0 \quad \text { for all } \quad u \in U, \quad \text { and } \quad i=1, \cdots, d .
$$

In (1.2), the initial data $u_{0}=\left(u_{0}^{1}, \cdots, u_{0}^{d}\right)$ is assumed to satisfy the following property:

$$
(H 3) \quad\left\{\begin{array}{l}
\alpha^{i} \leq u_{0}^{i} \leq \beta^{i}, \\
u_{0}^{i} \text { is nondecreasing, } \\
\partial_{x} u_{0}^{i} \in L \log L(\mathbb{R}),
\end{array} \mid \text { for } i=1, \cdots, d,\right.
$$

where $L \log L(\mathbb{R})$ is the following Zygmund space:

$$
L \log L(\mathbb{R})=\left\{f \in L^{1}(\mathbb{R}) \text { such that } \int_{\mathbb{R}}|f| \ln (e+|f|)<+\infty\right\} .
$$

This space is equipped by the following norm:

$$
\|f\|_{L \log L(\mathbb{R})}=\inf \left\{\mu>0: \int_{\mathbb{R}} \frac{|f|}{\mu} \ln \left(1+\frac{|f|}{\mu}\right) \leq 1\right\} .
$$

This norm is due to Luxemburg (see Adams [1], (13), Page 234]). 
In particular we will say that $u_{0}$ is nondecreasing if each component $u_{0}^{i}$, for $i=1, \ldots, d$, is nondecreasing and we write it as $\partial_{x} u_{0} \geq 0$. Recall that nondecreasing solutions of the classical scalar Burgers equation $\partial_{t} u+\partial_{x}\left(\frac{u^{2}}{2}\right)=0$, do not develop shocks. Notice that assumption $(H 2)$ is a natural generalization of Burgers equation to systems.

For general $(d \times d)$ strictly hyperbolic systems, (including diagonal systems, like system (1.1)), Bianchini and Bressan proved in 国 a striking result of global existence and uniqueness of a solution assuming that the initial data has small total variation. Their existence result is a generalization of Glimm's result [12], proved in the case of conservation laws. Let us mention that an existence result has also been obtained by LeFloch and Liu [18, 19] in the non-conservative case. In this paper we are interested in existence and uniqueness result of a continuous solution to system (1.1).

\subsection{Main results}

In El Hajj, Monneau [11], we left open the question of the uniqueness of continuous solutions of system (1.1). In this subsection we present two uniqueness results for system (1.1) under some particular assumptions. An application of these two main results is then presented in Subsection 1.3 for the $1 D$ gas dynamics equations.

Theorem 1.1 (Existence and uniqueness of a continuous solution) Assume (H1), (H2), (H3) and that system (1.1) is strictly hyperbolic, i.e.

$$
\lambda^{i+1}(u)-\lambda^{i}(u) \geq \Lambda>0, \quad \text { for all } u \in U \text { and } i=1, \ldots, d-1 .
$$

Then, there exists a function $u=\left(u^{i}\right)_{i=1, \ldots, d}$ which satisfies:

\section{i) Existence of a continuous solution:}

The function $u$ is solution of (1.1)-(1.8), such that $u(t, \cdot)$ is nondecreasing in $x$ for all $t>0$, $u(t, x) \in U$ for all $(t, x)$, and $u$ satisfies

$$
u \in\left[L^{\infty}((0,+\infty) \times \mathbb{R})\right]^{d} \text { and } \partial_{x} u \in\left[L^{\infty}((0,+\infty) ; L \log L(\mathbb{R}))\right]^{d} .
$$

Moreover $u$ is continuous in time and in space and satisfies for all $\delta, h \geq 0$ and all $(t, x) \in$ $(0, T-\delta) \times \mathbb{R}$, the following estimate:

$$
|u(t+\delta, x+h)-u(t, x)| \leq C \omega(\delta, h) \text { with } \omega(\delta, h)=\frac{1}{\ln \left(\frac{1}{\delta}+1\right)}+\frac{1}{\ln \left(\frac{1}{h}+1\right)},
$$

where $C\left(T, d, M_{0}, M_{1},\left\|u_{0}\right\|_{\left[L^{\infty}(\mathbb{R})\right]^{d}},\left\|\partial_{x} u_{0}\right\|_{[L \log L(\mathbb{R})]^{d}}, \Lambda\right)$.

Furthermore $u$, is a continuous vanishing viscosity solution of system (1.1)-(1.7), in the sense of Definition 3.0 .

\section{ii) Uniqueness:}

Under assumptions $(H 1),(H 2),(H 3)$ and (1.4) every continuous vanishing viscosity solution of (1.1)-(1.9) in the sense of Definition 3.0 is unique. 


\section{iii) $L^{1}$-stability estimate:}

Let $u$ (resp. v) be two solutions of system (1.1), constructed in (i). Assume moreover that $u(0, \cdot)=u_{0}(\cdot)$ and $v(0, \cdot)=v_{0}(\cdot)$ such that $u_{0}( \pm \infty)=v_{0}( \pm \infty)$. Then there exists a constant $L>0$, such that for all $t \in[0, T]$, we have

$$
\|u(t, \cdot)-v(t, \cdot)\|_{\left[L^{1}(\mathbb{R})\right]^{d}} \leq L\left\|u_{0}-v_{0}\right\|_{\left[L^{1}(\mathbb{R})\right]^{d}}
$$

where $L$ only depends on $T, M_{0}, M_{1}, d, \Lambda$ and bounds on $\left\|u_{0}\right\|_{\left[L^{\infty}(\mathbb{R})\right]^{d}},\left\|\partial_{x} u_{0}\right\|_{[L \log L(\mathbb{R})]^{d}}$, $\left\|v_{0}\right\|_{\left[L^{\infty}(\mathbb{R})\right]^{d}},\left\|\partial_{x} v_{0}\right\|_{[L \log L(\mathbb{R})]^{d}}$.

\section{Remark 1.2}

(i) Notice that if $u_{0} \in\left[W^{1, \infty}(\mathbb{R})\right]^{d}$ with $\partial_{x} u_{0} \geq 0$ then $\partial_{x} u_{0} \in\left(L^{1}(\mathbb{R}) \cap L^{\infty}(\mathbb{R})\right)^{d} \subset[L \log L(\mathbb{R})]^{d}$ and we can apply Theorem 1.1 .

(ii) If we know moreover that the system is rich then by a result of Serre [20, Vol II], we know that the solution is indeed Lipschitz. Therefore our Theorem 1.1 can be seen as a generalization of the result of Serre to the case of diagonal non-rich systems.

(iii) The $C^{\infty}$ regularity of the coefficients is convenient for the proofs, but can be weakened up to the minimal regularity, i.e. Lipschitz continuous coefficients $\lambda^{i}$.

Let us mention that a global existence result similar to Theorem 1.1 (but without uniqueness) has been obtained in [11] for non strictly hyperbolic systems where assumptions (H2)-(1.4) are simply replaced by the following assumption

$$
(H 2)^{\prime} \mid \begin{aligned}
& \text { for all } u \in U, \quad \text { we have } \\
& \sum_{i, j=1, \ldots, d} \xi_{i} \xi_{j} \lambda_{, j}^{i}(u) \geq 0 \quad \text { for every } \xi=\left(\xi_{1}, \ldots, \xi_{d}\right) \in[0,+\infty)^{d} .
\end{aligned}
$$

Notice that in the case of strictly hyperbolic systems, Theorem 1.1 only requires assumption (H2) which is weaker than $(H 2)^{\prime}$ and moreover guarantees the uniqueness of the solution. Our method of proof is strongly inspired from Bianchini, Bressan [4]. First, we get an estimate in $\left[L^{\infty}((0, T) ; L \log L(\mathbb{R}))\right]^{d}$ for $\partial_{x} u$ getting some control on the interactions between different fields $\int_{0}^{T} \int_{\mathbb{R}} \partial_{x} u^{i} \partial_{x} u^{j} d x d t$ for $i \neq j$, using the strictly hyperbolic condition (1.4) similarly as in Bianchini et al. [4].

A second key point is that our $\left[L^{\infty}((0, T) ; L \log L(\mathbb{R}))\right]^{d}$ estimate on $\partial_{x} u$ implies the continuity of the solution $u$ with a controlled modulus of continuity. This implies that the solution is locally in $B V$ with small norm. Taking into account the finite speed propagation property it is then possible to localize the argument developed in Bianchini et al. 团, and finally to extend it to the case of large initial data (but monotone data).

Let us mention that, in the case $d=2$ and under the same assumptions of Theorem 1.1, T. T. Li proved in [20, pp. 35-41] an existence and uniqueness result for $C^{1}$ solutions. This result is a generalization of Lax result [17, proved for Lipschitz solutions. Here, we prove a similar result considering less regularity on the solution (continuous solutions) and for all $d \geq 1$. 
Let us now introduce various assumptions on the matrix $\left(\lambda_{, j}^{i}(u)\right)_{i, j=1, \ldots, d}$ which will guarantee the existence and uniqueness of Lipschitz solutions.

(Non-negative sub-diagonal matrices)

$$
\lambda_{, j}^{i}(u) \geq 0 \quad \text { for all } \quad u \in U \text { and } \quad j \geq i \quad \text { with } \quad i, j \in\{1, \ldots, d\}
$$

\section{(Non-negative matrices with non-positive off-diagonal terms)}

$$
\left\{\begin{array}{l}
\lambda_{, j}^{i}(u) \leq 0 \quad \text { for all } u \in U \text { and } j \neq i \quad \text { with } i, j \in\{1, \ldots, d\} \\
A_{i j}=\inf _{u \in U}\left(\lambda_{, j}^{i}(u)\right) \text { and } \sum_{i, j=1, \ldots, d} A_{i j} \xi_{i} \xi_{j} \geq 0 \text { for every } \xi=\left(\xi_{1}, \ldots, \xi_{d}\right) \in[0,+\infty)^{d} .
\end{array}\right.
$$

\section{(Diagonally dominant)}

$$
\lambda_{, i}^{i}(u) \geq \sum_{i \neq j}\left(\lambda_{, j}^{i}(u)\right)^{-} \quad \text { for all } \quad u \in U \quad \text { and } \quad i=1, \ldots, d
$$

where we note $x^{-}=\max (0,-x)$.

\section{Theorem 1.3 (Existence and uniqueness of Lipschitz solutions)}

Assume one of the following assumptions $(K 1)$, (K2) or $(K 3)$. Let $u_{0} \in\left[W^{1, \infty}(\mathbb{R})\right]^{d}$ be a nondecreasing function satisfying $u_{0}(x) \in U$, for all $x \in \mathbb{R}$. Then, there exists a unique function $u \in \bigcap_{T>0}\left[W^{1, \infty}([0, T) \times \mathbb{R})\right]^{d}$ solution of 1.1)-(1.9), with $u(t, x) \in U$ for all $(t, x)$. Moreover we have for any $t \in(0,+\infty)$ :

$$
\sum_{i=1, \ldots, d}\left\|\partial_{x} u^{i}(t, \cdot)\right\|_{L^{\infty}(\mathbb{R})} \leq \sum_{i=1, \ldots, d}\left\|\partial_{x} u_{0}^{i}\right\|_{L^{\infty}(\mathbb{R})}, \quad \text { if } \quad(K 2) \quad \text { holds }
$$

and

$$
\max _{i=1, \ldots, d}\left\|\partial_{x} u^{i}(t, \cdot)\right\|_{L^{\infty}(\mathbb{R})} \leq \max _{i=1, \ldots, d}\left\|\partial_{x} u_{0}^{i}\right\|_{L^{\infty}(\mathbb{R})}, \quad \text { if } \quad(K 3) \quad \text { holds. }
$$

Notice that in Theorem 1.3, we do not assume that system (1.1) is strictly hyperbolic.

Theorem 1.3 is based on the fact that the solution satisfies $\partial_{x} u^{i} \geq 0$, for $i=1, \ldots, d$, and then we only have to bound the maximum of the gradient from one side. Assumptions $(K 1),(K 2)$ and $(K 3)$ are sufficient conditions to control the solution of the maximum of the gradient. These a priori bounds are obtained considering a parabolic regularization of the system and then writing some differential inequalities satisfied in the sense of viscosity by the maximum of the gradient. The uniqueness of the solution is an independent result valid for Lipschitz solutions.

In the case of $(2 \times 2)$ strictly hyperbolic systems, which corresponds in (1.1) to the case of $\lambda^{1}\left(u^{1}, u^{2}\right)<\lambda^{2}\left(u^{1}, u^{2}\right)$, we refer the reader to the work of Lax [17, which has proved the 
existence of Lipschitz solutions of (1.1)-(1.2) with the assumption $\lambda_{, i}^{i}(u) \geq 0$ for the diagonal terms. As it was recalled in Remark 1.2 (ii), this result was also extended by Serre 26, Vol II] to the case of $(d \times d)$ rich strictly hyperbolic systems. We also refer the reader to the work of Poupaud [25], for a global existence and uniqueness result of a Lipschitz solution of a particular quasi-linear hyperbolic system, considering large initial data.

In the framework of viscosity solutions, Ishii, Koike [15] and Ishii [14], have shown existence and uniqueness of viscosity continuous solutions for Hamilton-Jacobi systems of the form:

$$
\begin{cases}\partial_{t} u^{i}+H_{i}\left(u, D u^{i}\right)=0 & \text { with } u=\left(u^{1}, \ldots, u^{d}\right) \in \mathbb{R}^{d}, \text { for } x \in \mathbb{R}^{N}, t \in(0,+\infty), \\ u^{i}(x, 0)=u_{0}^{i}(x) & x \in \mathbb{R}^{N},\end{cases}
$$

where the Hamiltonian $H_{i}$ is quasi-monotone in $u$ (see the definition in Ishii, Koike 15, Th.4.7]). Indeed system (1.1) belongs to this framework with $N=1$ and $\partial_{x} u^{i} \geq 0$ under the assumption $\lambda_{, j}^{i}(u) \leq 0$ for $j \neq i$.

Let us also mention that in the case $d=2$ with a matrix $\left(\lambda_{, j}^{i}(u)\right)_{i, j=1,2}=\left(\begin{array}{cc}1 & -1 \\ -1 & 1\end{array}\right)$, it was proved in El Hajj, Forcadel [10], the existence and uniqueness of a Lipschitz viscosity solution, and in El Hajj [9], the existence and uniqueness of a strong solution in $\left[W_{l o c}^{1,2}([0,+\infty) \times \mathbb{R})\right]^{2}$.

\subsection{Application to $1 D$ gas dynamics}

Now, we present an application of the previous results to the following $1 D$ system of isentropic gas dynamics:

$$
\left\{\begin{array}{l}
\partial_{t} \rho+\partial_{x}(\rho u)=0 \\
\partial_{t}(\rho u)+\partial_{x}\left(\rho u^{2}+p(\rho)\right)=0, \quad \text { with } \quad p(\rho)=\frac{(\gamma-1)^{2}}{4 \gamma} \rho^{\gamma} \\
u(0, x)=u_{0} \quad \text { and } \quad \rho(0, x)=\rho_{0} \geq 0 .
\end{array} \mid \text { on } \quad(0,+\infty) \times \mathbb{R}\right.
$$

where $\rho$ is the density, $u$ is the speed and $p(\rho)$ is the pressure given by a simple power law for an exponent $\gamma>1$. First, we assume the following conditions, with $\theta=\frac{\gamma-1}{2}$ :

$(J 1) \quad u_{0}, \rho_{0}^{\theta} \in L^{\infty}(\mathbb{R}), \quad$ and $\quad \partial_{x} u_{0} \geq\left|\partial_{x} \rho_{0}^{\theta}\right|$.

$(J 2) \quad \partial_{x} u_{0}, \partial_{x} \rho_{0}^{\theta} \in L \log L(\mathbb{R})$.

$(J 2)^{\prime} \quad u_{0}, \rho_{0}^{\theta} \in \operatorname{Lip}(\mathbb{R})$.

Applying Theorems 1.1 and 1.3, we will prove the following result.

Theorem 1.4 (Existence and uniqueness for isentropic gas dynamics) Assume (J1), with $\rho_{0} \geq 0$ and $\gamma>1$. Then we have 
i) Existence and uniqueness of a continuous solution:

Under assumption $(J 2)$, system (1.10) has a continuous solution $(\rho, u)$ on $[0,+\infty) \times \mathbb{R}$, where $\rho(t, \cdot)$ and $u(t, \cdot)$ satisfy $(J 1)$ and $(J 2)$, for all $t \geq 0$. Moreover, if

$$
u_{0}+\rho_{0}^{\theta} \geq \Lambda_{1}>\Lambda_{2} \geq u_{0}-\rho_{0}^{\theta} \quad \text { for some constants } \Lambda_{1}, \Lambda_{2},
$$

then this solution is the unique continuous vanishing viscosity solution, in the sense of Definition 3.9.

ii) Existence and uniqueness of a Lipschitz solution:

Assume $(J 2)^{\prime}$. If $1<\gamma \leq 3$, then system (1.10) has a solution

$(\rho, u) \in\left[L^{\infty}([0,+\infty) \times \mathbb{R})\right]^{2}$, with

$$
\rho \geq 0 \quad \text { and } \quad \rho^{\theta}, u \in W^{1, \infty}([0,+\infty) \times \mathbb{R}) .
$$

Reciprocally any solution $(\rho, u)$ of (1.10) satisfying (1.11) is unique if we assume moreover that $\rho \geq \Lambda>0$ on $[0,+\infty) \times \mathbb{R}$.

\section{Remark 1.5 (Vacuum case)}

Notice that if $\rho=0$ on a subset $\omega \subset(0,+\infty) \times \mathbb{R}$, then equation (1.10) is automatically satisfied and the function $u$ can be chosen locally arbitrarily in $\omega$. This shows that we can not expect uniqueness of the solution when there is vacuum (i.e $\rho=0$ ).

The proof of Theorem 1.4 is an application of Theorems 1.1 and 1.3. We refer the reader to Section 5 for the proof of Theorem 1.4. Let us recall that, in the case $\rho_{0}>0$, T. T. Li proved in [20, pp. 35-41] an existence and uniqueness result for $C^{1}$ solutions. Notice that for the existence results given in (i) (continuous solutions) and in (ii) (Lipschitz solutions), we only assume that $\rho_{0} \geq 0$, which allows us to consider solutions with vacuum. In connection with Theorem 1.4, let us mention the work of Lions et al. in [22] where the existence of a solution was obtained for $\rho_{0} \geq 0$ with any $u_{0}, \rho_{0} \in L^{\infty}(\mathbb{R})$ and $\gamma>1$. This extended a previous result of DiPerna [7, 8]. We also refer the reader to Mercier [23] for another result with vacuum.

\subsection{Organization of the paper}

This paper is organized as follows: in Section 2, we prove the existence of continuous solutions (Theorem 1.1 $(i)$ ). In Section 3, we prove the uniqueness of continuous vanishing viscosity solutions (Theorem 1.1 (ii)) and the $L^{1}$-stability estimate (Theorem 1.1 (iii)). In Section 1 , we prove the existence and uniqueness of Lipschitz solutions (Theorem 1.3 . $)$. Finally in Section 5, we give the proof of Theorem 1.4 as an application to the $1 D$ isentropic gas dynamics.

\section{Existence of continuous solutions}

In this section we prove the existence of continuous solutions of system (1.1)-(1.2) (Theorem $1.1(i)$ ) adapting our existence proof developed in 11] and some ideas of Bianchini, Bressan 伆.

To prove the existence of continuous solutions to system (1.1)-(1.2), we need to recall the existence result proved by El Hajj et al. in [11] for the following parabolic regularization of system (1.1)-(1.2): 


$$
\left\{\begin{array}{l}
\partial_{t} u^{i, \varepsilon}+\lambda^{i}\left(u^{\varepsilon}\right) \partial_{x} u^{i, \varepsilon}=\varepsilon \partial_{x x} u^{i, \varepsilon} \quad \text { with } \quad 0<\varepsilon \leq 1 \quad \text { and } \quad \partial_{x x}=\frac{\partial^{2}}{\partial x^{2}} \\
u^{\varepsilon}(x, 0)=u_{0}^{\varepsilon}(x), \quad \text { with } \quad u_{0}^{\varepsilon}(x):=u_{0} * \eta_{\varepsilon}(x),
\end{array}\right.
$$

where we have also regularized the initial data by convolution with a mollifier $\eta_{\varepsilon}$ defined by $\eta_{\varepsilon}(\cdot)=\frac{1}{\varepsilon} \eta(\dot{\bar{\varepsilon}})$, for some non-negative function $\eta \in C_{c}^{\infty}(\mathbb{R})$ satisfying $\int_{\mathbb{R}} \eta=1$.

We will need to use (and to prove later) the following assumption:

$$
\mid \begin{aligned}
& \text { For all } T>0, \quad \exists C_{T}>0, \quad \text { such that } \\
& \left\|\partial_{x} u^{i, \varepsilon}\right\|_{L^{\infty}((0, T) ; L \log L(\mathbb{R}))} \leq C_{T} \text { for } i=1, \ldots, d .
\end{aligned}
$$

In [11], we have proven the following result (see [11], Theorem 2.2, Proposition 3.1, Lemma 4.3 and Theorem 4.4).

Theorem 2.1 (Global existence for non strictly hyperbolic case) Assume (H1) and (H3). Then we have:

\section{i) Existence:}

There exists a function $u^{\varepsilon}=\left(u^{i, \varepsilon}\right)_{i=1, \ldots, d} \in\left[C^{\infty}([0,+\infty) \times \mathbb{R})\right]^{d}$ solution of (2.1), such that the function $u^{\varepsilon}(t, \cdot)$ is nondecreasing in $x$ for all $t>0, u^{\varepsilon}(t, x) \in U$ for all $(t, x)$, and $u^{\varepsilon}$ satisfies the following $L^{\infty}$ estimate:

$$
\left\|u^{i, \varepsilon}\right\|_{L^{\infty}((0,+\infty) \times \mathbb{R})} \leq\left\|u_{0}^{i}\right\|_{L^{\infty}(\mathbb{R})} \quad \text { for } \quad i=1, \ldots, d .
$$

Moreover, we have $u^{\varepsilon} \in \bigcap_{T>0}\left[W^{2, \infty}([0, T) \times \mathbb{R})\right]^{d}$ and we have for any $t \in[0, T]$ the following gradient entropy estimate:

$$
\int_{\mathbb{R}} \sum_{i=1, \ldots, d} f\left(\partial_{x} u^{i, \varepsilon}(t, x)\right) d x+\int_{0}^{t} \int_{\mathbb{R}_{i, j=1, \ldots, d}} \lambda_{, j}^{i}\left(u^{\varepsilon}\right) \partial_{x} u^{i, \varepsilon}(s, x) \partial_{x} u^{j, \varepsilon}(s, x) d x d s \leq C_{1},
$$

where

$$
f(x)= \begin{cases}x \ln (x)+\frac{1}{e} & \text { if } \quad x \geq 1 / e \\ 0 & \text { if } \quad 0 \leq x \leq 1 / e,\end{cases}
$$

and $C_{1}\left(T, d, M_{1},\left\|u_{0}\right\|_{\left[L^{\infty}(\mathbb{R})\right]^{d}},\left\|\partial_{x} u_{0}\right\|_{[L \log L(\mathbb{R})]^{d}}\right)$.

\section{ii) Convergence:}

Assume moreover that $u^{\varepsilon}$ satisfies $(A)$ uniformly for $\varepsilon \in(0,1]$. Then up to extract a subsequence, the function $u^{\varepsilon}$ converges locally uniformly, as $\varepsilon$ goes to zero, to a function $u \in\left[L^{\infty}([0,+\infty) \times \mathbb{R})\right]^{d}$. Moreover $u$ is a solution to (1.1)-(1.A) and satisfies $u(t, \cdot)$ is nondecreasing in $x$ for all $t>0, u \in[C([0,+\infty) \times \mathbb{R})]^{d}, u(t, x) \in U$ for all $(t, x)$ and there exists a modulus of continuity $\omega(\delta, h)$, such that for all $\delta, h \geq 0$ and all $(t, x) \in(0, T-\delta) \times \mathbb{R}$, we have:

$$
|u(t+\delta, x+h)-u(t, x)| \leq C_{2} \omega(\delta, h) \text { with } \omega(\delta, h)=\frac{1}{\ln \left(\frac{1}{\delta}+1\right)}+\frac{1}{\ln \left(\frac{1}{h}+1\right)}
$$


where $C_{2}\left(C_{T}, M_{0}\right)$, with $C_{T}$ is given in assumption $(A)$.

Before going into the proof of the existence result of continuous solutions introduced in Theorem

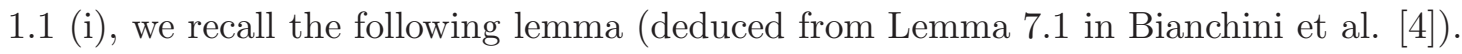

\section{Lemma 2.2 (Transversal wave interactions)}

Let $\mu, \bar{\mu} \in C_{b}((0,+\infty) \times \mathbb{R})$ (two continuous bounded functions) and $\varepsilon \geq 0$. Let moreover $z, \bar{z} \in L^{\infty}\left((0,+\infty) ; L^{1}(\mathbb{R})\right)$, be solutions of the two independent scalar equations

$$
\begin{aligned}
& \partial_{t} z+\partial_{x}(\mu z)=\varepsilon \partial_{x x} z \quad \text { on } \quad(0,+\infty) \times \mathbb{R} \\
& \partial_{t} \bar{z}+\partial_{x}(\bar{\mu} \bar{z})=\varepsilon \partial_{x x} \bar{z} \quad \text { on } \quad(0,+\infty) \times \mathbb{R}
\end{aligned}
$$

with two initial data $z(0, \cdot), \bar{z}(0, \cdot) \in L^{1}(\mathbb{R})$, where the initial data of $z$ is understood as follows

$$
\int_{\mathbb{R}} z(t, x) \psi(x) d x \rightarrow \int_{\mathbb{R}} z(0, x) \psi(x) d x \quad \text { as } \quad t \rightarrow 0, \quad \text { for every } \quad \psi \in C_{c}^{\infty}(\mathbb{R})
$$

and similarly for $\bar{z}$. Assume that, for all $T>0$

$$
\inf _{(t, x) \in(0, T) \times \mathbb{R}}[\mu(t, x)-\bar{\mu}(t, x)] \geq \Lambda>0 .
$$

Then

$$
\int_{0}^{T} \int_{\mathbb{R}}|z(t, x)||\bar{z}(t, x)| d x d t \leq \frac{1}{\Lambda}\left(\int_{\mathbb{R}}|z(0, x)| d x\right)\left(\int_{\mathbb{R}}|\bar{z}(0, x)| d x\right) .
$$

We remark that the proof of this lemma is based on the following estimate

$$
\frac{d}{d t}\left[\iint_{x<y} \frac{1}{\Lambda}|z(t, x) \| \bar{z}(t, y)| d x d y\right] \leq-\int_{\mathbb{R}}|z(t, x)||\bar{z}(t, x)| d x .
$$

For more details see Bianchini et al. [4, Lemma 7.1].

Proof of Theorem 1.1 (i):

We will show that bound $(A)$ holds for the solution $u^{\varepsilon}$ given in Theorem 2.1 (i). To this end, we bound from above the following quantity uniformly on $\varepsilon$

$$
\begin{aligned}
I & =-\int_{0}^{t} \int_{\mathbb{R}} \sum_{i, j=1, \ldots, d} \lambda_{, j}^{i}\left(u^{\varepsilon}\right) \partial_{x} u^{\varepsilon, i}(s, x) \partial_{x} u^{\varepsilon, j}(s, x) d x d s \\
& \leq-\int_{0}^{t} \int_{\mathbb{R}} \sum_{i \neq j, i, j=1, \ldots, d} \lambda_{, j}^{i}\left(u^{\varepsilon}\right) \partial_{x} u^{\varepsilon, i}(s, x) \partial_{x} u^{\varepsilon, j}(s, x) d x d s \\
& \leq M_{1} \int_{0}^{t} \int_{\mathbb{R}} \sum_{i \neq j, i, j=1, \ldots, d}\left|\partial_{x} u^{\varepsilon, i}(s, x)\right|\left|\partial_{x} u^{\varepsilon, j}(s, x)\right| d x d s
\end{aligned}
$$


where to get the second line we have used $(H 2)$, and we have used $(H 1)$ in the third line. Now, we use (1.4), Lemma 2.2 and the monotonicity of $u_{0}^{\varepsilon}$ (as a consequence of (H3)), we obtain

$$
\begin{aligned}
I & \leq \frac{M_{1}}{\Lambda} \sum_{i \neq j, i, j=1, \ldots, d}\left(\int_{\mathbb{R}}\left|\partial_{x} u_{0}^{\varepsilon, i}(x)\right| d x\right)\left(\int_{\mathbb{R}}\left|\partial_{x} u_{0}^{\varepsilon, j}(x)\right| d x\right) \\
& \leq \frac{4 M_{1}}{\Lambda}\left\|u_{0}\right\|_{\left[L^{\infty}(\mathbb{R})\right]^{d}}^{2} .
\end{aligned}
$$

Then, by (2.3) we get

$$
\int_{\mathbb{R}} \sum_{i=1, \ldots, d} f\left(\partial_{x} u^{\varepsilon, i}(t, x)\right) d x \leq C_{1}+\frac{4 M_{1}}{\Lambda}\left\|u_{0}\right\|_{\left[L^{\infty}(\mathbb{R})\right]^{d}}^{2}:=C_{T},
$$

which implies that $\partial_{x} u^{\varepsilon, i}$, for $i=1, \ldots, d$, are bounded in $\left[L^{\infty}((0, T) ; L \log L(\mathbb{R}))\right]^{d}$ uniformly on $\varepsilon$ (with a constant only depending on $C_{T}$ and on $\left\|u_{0}\right\|_{\left[L^{\infty}(\mathbb{R})\right]^{d}}$ ).

The fact that $u$ is a vanishing viscosity solution is a consequence of Theorem 3.7 that will be proven later. This ends the proof of Theorem 1.1 (i).

\section{Local semigroup property and uniqueness of continuous van- ishing viscosity solutions}

In this section, we show that the solution of system (1.1)-(1.2), constructed in Theorem 1.1 (i), is the unique continuous vanishing viscosity solution (in the sense of Definition 3.6). In the following subsection we show some useful estimates for the parabolic system (2.1). Then using these estimates, we prove in Subsection 3.2 a kind of "finite propagation speed result" of this parabolic system in the vanishing viscosity limit. Thanks to this result, we are able to localize the argument developed in Bianchini et al. [4] and then to extend it for large and continuous data.

\subsection{Preliminary results}

In this subsection we show some useful parabolic estimates. In Proposition 3.2, we prove that the $L^{1}$ norm of the second space derivative $u_{x x}$ of the solution of parabolic system (2.1) decays rapidly in space locally in time, which gives a $L^{\infty}$ bound on the space derivative $u_{x}$. Then, using this $L^{\infty}$ bound we prove in Lemma 3.4 a comparison principle result based on the maximum principle for scalar parabolic equations.

\section{Lemma 3.1 (Properties of the heat kernel)}

Let $G(t, x)=\frac{1}{\sqrt{4 \pi t}} e^{-\frac{x^{2}}{4 t}}$ be the standard heat kernel. Then, for all $t>0$, we have:

(i) $\|G(t, \cdot)\|_{L^{1}(\mathbb{R})}=1$,

(ii) $\left\|\partial_{x} G(t, \cdot)\right\|_{L^{1}(\mathbb{R})} \leq \frac{1}{\sqrt{t}}$. 
For the proof of this lemma, we refer to Pazy [24, Th 5.2. Page 107].

Proposition 3.2 (Local in time $L^{1}$ bound on $\partial_{x x} u^{\varepsilon}$ )

Let $u^{\varepsilon}=\left(u^{\varepsilon, i}\right)_{i=1, \ldots, d}$ be the solution of system (2.1), given by Theorem 2.1 (i). Then for

$$
T_{0}=\left(\frac{1}{8 C_{0}}\right)^{2} \quad \text { and } \quad C_{0}=2\left(\left\|u_{0}\right\|_{\left[L^{\infty}(\mathbb{R})\right]^{d}}+M_{0}+M_{1}\left\|u_{0}\right\|_{\left[L^{\infty}(\mathbb{R})\right]^{d}}\right),
$$

the following estimate holds for all $t \in\left[0, \varepsilon T_{0}\right]$ :

$$
\left\|\partial_{x x} u^{\varepsilon, i}(t, \cdot)\right\|_{L^{1}(\mathbb{R})} \leq \frac{2 C_{0}}{\sqrt{\varepsilon t}} \quad \text { for } \quad i=1, \ldots, d .
$$

\section{Proof of Proposition 3.2:}

We prove the result in three steps. In the first step, we prove that the second space derivative of the solution of (2.1) with $\varepsilon=1$ is bounded in $L^{\infty}\left((0, T) ; L^{1}(\mathbb{R})\right)$ for some small $T$. In the second step we prove estimate (3.1) in the case $\varepsilon=1$ and then in the third step we deduce the result rescaling in time and in space.

Step 1. (Local $L^{\infty}\left((0, T) ; L^{1}(\mathbb{R})\right)$ bound): Let $v=\left(v^{i}\right)_{i=1, \ldots, d}$ be a solution of system (2.1), with $\varepsilon=1$, given by Theorem 2.1 (i). Taking the derivative with respect to $x$ the equation (2.1) satisfied by $v \in\left[C^{\infty}((0, T) \times \mathbb{R})\right]^{d}$, we get that $w^{i}=\partial_{x} v^{i}$ satisfies the following equation

$$
\partial_{t} w^{i}+\lambda^{i}(v) \partial_{x} w^{i}+\sum_{j=1, \ldots, d} \lambda_{, j}^{i}(v) w^{j} w^{i}=\partial_{x x} w^{i} .
$$

The function $w^{i}(t)=w^{i}(t, \cdot)$, can be represented as

$$
w^{i}(t)=G(t) * w^{i}(0)-\int_{0}^{t} G(t-s) *\left[\lambda^{i}(v) \partial_{x} w^{i}+\sum_{j=1, \ldots, d} \lambda_{, j}^{i}(v) w^{j} w^{i}\right] d s
$$

where $G$ is defined in Lemma 3.1. Taking the derivative with respect to $x$ we deduce that

$$
\partial_{x} w^{i}(t)=G(t) * \partial_{x} w^{i}(0)-\int_{0}^{t}\left(\partial_{x} G(t-s)\right) *\left[\lambda^{i}(v) \partial_{x} w^{i}+\sum_{j=1, \ldots, d} \lambda_{, j}^{i}(v) w^{j} w^{i}\right] d s .
$$

Using Lemma 3.1, we obtain

$$
\begin{aligned}
\left\|\partial_{x} w^{i}(t)\right\|_{L^{1}(\mathbb{R})} \leq\left\|\partial_{x} w^{i}(0)\right\|_{L^{1}(\mathbb{R})} & +M_{0} \int_{0}^{t} \frac{1}{\sqrt{t-s}}\left\|\partial_{x} w^{i}(s)\right\|_{L^{1}(\mathbb{R})} d s \\
& +M_{1} \int_{0}^{t} \frac{1}{\sqrt{t-s}}\left\|w^{i}(s)\right\|_{L^{\infty}(\mathbb{R})}\|w(s)\|_{\left[L^{1}(\mathbb{R})\right]^{d}} d s
\end{aligned}
$$

where $w=\left(w^{i}\right)_{i=1, \ldots, d}$. Using estimate (2.2) and the fact that $w^{i} \geq 0$, we obtain 


$$
\begin{aligned}
\left\|\partial_{x} w^{i}(t)\right\|_{L^{1}(\mathbb{R})} \leq\left\|\partial_{x} w^{i}(0)\right\|_{L^{1}(\mathbb{R})} & +M_{0} \int_{0}^{t} \frac{1}{\sqrt{t-s}}\left\|\partial_{x} w^{i}(s)\right\|_{L^{1}(\mathbb{R})} d s \\
& +2 M_{1}\left\|u_{0}\right\|_{\left[L^{\infty}(\mathbb{R})\right]^{d}} \int_{0}^{t} \frac{1}{\sqrt{t-s}}\left\|w^{i}(s)\right\|_{L^{\infty}(\mathbb{R})} d s .
\end{aligned}
$$

By Sobolev injection and the fact that $w^{i}(t, x) \rightarrow 0$ as $|x| \rightarrow+\infty$, we can see that

$$
\begin{aligned}
\left\|\partial_{x} w^{i}(t)\right\|_{L^{1}(\mathbb{R})} \leq\left\|\partial_{x} w^{i}(0)\right\|_{L^{1}(\mathbb{R})} & +M_{0} \int_{0}^{t} \frac{1}{\sqrt{t-s}}\left\|\partial_{x} w^{i}(s)\right\|_{L^{1}(\mathbb{R})} d s \\
& +2 M_{1}\left\|u_{0}\right\|_{\left[L^{\infty}(\mathbb{R})\right]^{d}} \int_{0}^{t} \frac{1}{\sqrt{t-s}}\left\|\partial_{x} w^{i}(s)\right\|_{L^{1}(\mathbb{R})} d s .
\end{aligned}
$$

This implies that

$$
\left\|\partial_{x} w^{i}(t)\right\|_{L^{1}(\mathbb{R})} \leq\left\|\partial_{x} w^{i}(0)\right\|_{L^{1}(\mathbb{R})}+\kappa \sqrt{T}\left\|\partial_{x} w^{i}\right\|_{L^{\infty}\left((0, T) ; L^{1}(\mathbb{R})\right)}
$$

where $\kappa=2\left(M_{0}+2 M_{1}\left\|u_{0}\right\|_{\left.\left[L^{\infty}(\mathbb{R})\right]^{d}\right)}\right.$. Using estimate (3.4), we can prove that for all $T \leq \frac{1}{4 \kappa^{2}}$, we have (if $\left\|\partial_{x} w^{i}\right\|_{L^{\infty}\left((0, T) ; L^{1}(\mathbb{R})\right)}$ is finite)

$$
\left\|\partial_{x} w^{i}\right\|_{L^{\infty}\left((0, T) ; L^{1}(\mathbb{R})\right)} \leq 2\left\|\partial_{x} w^{i}(0)\right\|_{L^{1}(\mathbb{R})}=2\left\|\partial_{x x}\left(u_{0}^{i} * \eta_{1}\right)\right\|_{L^{1}(\mathbb{R})} .
$$

The remaining difficulty is to show that $\left\|\partial_{x} w^{i}\right\|_{L^{\infty}\left((0, T) ; L^{1}(\mathbb{R})\right)}$ is finite. To this end, we multiply $w$ by a function $\phi_{R}(\cdot)=\phi(\dot{\bar{R}})$, where $\phi$ is a cut-off function satisfying $\phi \in C_{c}(\mathbb{R})$ and $\phi \equiv 1$ on $[-1,1]$. Then, we repeat the previous argument replacing $w$ by $\phi_{R} w$ and at the end we take the limit $R \rightarrow+\infty$ to conclude.

Step 2. (Case $\varepsilon=1$ ): We now write the derivative of equation (3.3) with respect to $x$, as follows

$$
\partial_{x} w^{i}(t)=\left(\partial_{x} G(t)\right) * w^{i}(0)-\int_{0}^{t}\left(\partial_{x} G(t-s)\right) *\left[\lambda^{i}(v) \partial_{x} w^{i}+\sum_{j=1, \ldots, d} \lambda_{, j}^{i}(v) w^{j} w^{i}\right] d s
$$

Using Lemma 3.1, we obtain

$$
\begin{aligned}
\left\|\partial_{x} w^{i}(t)\right\|_{L^{1}(\mathbb{R})} \leq \frac{1}{\sqrt{t}}\left\|w^{i}(0)\right\|_{L^{1}(\mathbb{R})} & +M_{0} \int_{0}^{t} \frac{1}{\sqrt{t-s}}\left\|\partial_{x} w^{i}(s)\right\|_{L^{1}(\mathbb{R})} d s \\
& +M_{1} \int_{0}^{t} \frac{1}{\sqrt{t-s}}\left\|w^{i}(s)\right\|_{L^{\infty}(\mathbb{R})}\|w(s)\|_{\left[L^{1}(\mathbb{R})\right]^{d}} d s .
\end{aligned}
$$

Similarly as in Step 1, from estimate (2.2) and the fact that $w^{i} \geq 0$, we get

$$
\begin{aligned}
\left\|\partial_{x} w^{i}(t)\right\|_{L^{1}(\mathbb{R})} \leq \frac{2}{\sqrt{t}}\left\|u_{0}\right\|_{\left[L^{\infty}(\mathbb{R})\right]^{d}} & +M_{0} \int_{0}^{t} \frac{1}{\sqrt{t-s}}\left\|\partial_{x} w^{i}(s)\right\|_{L^{1}(\mathbb{R})} d s \\
& +2 M_{1}\left\|u_{0}\right\|_{\left[L^{\infty}(\mathbb{R})\right]^{d}} \int_{0}^{t} \frac{1}{\sqrt{t-s}}\left\|\partial_{x} w^{i}(s)\right\|_{L^{1}(\mathbb{R})} d s .
\end{aligned}
$$


If we note $C_{0}=2\left(\left\|u_{0}\right\|_{\left[L^{\infty}(\mathbb{R})\right]^{d}}+M_{0}+M_{1}\left\|u_{0}\right\|_{\left[L^{\infty}(\mathbb{R})\right]^{d}}\right)$, then we can deduce that

$$
\left\|\partial_{x} w^{i}(t)\right\|_{L^{1}(\mathbb{R})} \leq \frac{C_{0}}{\sqrt{t}}+C_{0} \int_{0}^{t} \frac{1}{\sqrt{t-s}}\left\|\partial_{x} w^{i}(s)\right\|_{L^{1}(\mathbb{R})} d s .
$$

To prove (3.1), we shall argue by contradiction. First, we remark that from Step 1 we know that $\left\|\partial_{x} w^{i}(t)\right\|_{L^{1}(\mathbb{R})}$ is finite. Assume that there exists a first time $\tau<T_{0}$ such that the equality in (3.1) (with $\varepsilon=1$ ) holds. Then, observing that

$$
\int_{0}^{t} \frac{1}{\sqrt{s(t-s)}} d s=\pi
$$

we compute

$$
\begin{aligned}
\left\|\partial_{x} w^{i}(\tau)\right\|_{L^{1}(\mathbb{R})} & \leq \frac{C_{0}}{\sqrt{\tau}}+C_{0} \int_{0}^{\tau} \frac{1}{\sqrt{\tau-s}} \frac{2 C_{0}}{\sqrt{s}} d s \\
& <\frac{C_{0}}{\sqrt{\tau}}+8 C_{0}^{2} \leq \frac{2 C_{0}}{\sqrt{\tau}}
\end{aligned}
$$

reaching a contradiction. Hence,

$$
\left\|\partial_{x} w^{i}(t)\right\|_{L^{1}(\mathbb{R})}<\frac{2 C_{0}}{\sqrt{t}} \quad \text { for all } \quad t \in\left[0, T_{0}\right] .
$$

Step 3. (Case $\varepsilon>0$ ): We remark that if $v$ is solution of system (2.1), with $\varepsilon=1$, then $\overline{u^{\varepsilon}}(t, x)=v\left(\frac{t}{\varepsilon}, \frac{x}{\varepsilon}\right)$ is a solution of system (2.1), with $\varepsilon>0$. Applying (3.5), we get the result.

Corollary 3.3 (Global $L^{1}$ bound on $\partial_{x x} u^{\varepsilon}$ )

Under the assumptions of Proposition 3.9, we have for all $t>0$, and for $i=1, \ldots, d$

$$
\left\|\partial_{x x} u^{\varepsilon, i}(t, \cdot)\right\|_{L^{1}(\mathbb{R})} \leq \begin{cases}\frac{2 C_{0}}{\sqrt{\varepsilon t}} & \text { if } t<\varepsilon T_{0} \\ \frac{2 C_{0}}{\sqrt{\varepsilon T_{0}}} & \text { if } t \geq \varepsilon T_{0}\end{cases}
$$

where $C_{0}$ is defined in Proposition 3.2.

To prove this Corollary it suffices to apply Proposition 3.2 on the time interval $\left[t-\varepsilon T_{0}, t\right]$.

\section{Lemma 3.4 (Exponential estimate)}

Let $u$ be the solution of system (2.1), with $\varepsilon=1$, given by Theorem 2.1 (i). We consider a solution $z=\left(z^{i}\right)_{i=1, \ldots, d}$ of the linearized system:

$$
\partial_{t} z^{i}+\partial_{x}\left(\lambda^{i}(u) z^{i}\right)-\partial_{x x} z^{i}=\sum_{j=1, \cdots, d} \lambda_{, j}^{i}(u)\left[z^{i} \partial_{x} u^{j}-z^{j} \partial_{x} u^{i}\right] \quad \text { for } \quad i=1, \cdots, d
$$

with initial data satisfying

$$
\begin{cases}|z(0, x)| \leq 1 & \text { if } \quad x \geq 0 \\ z(0, x)=0 & \text { if } \quad x<0\end{cases}
$$


Then, there exists two constants $\alpha, \beta>0$, such that for all $t>0$

$$
|z(t, x)| \leq \alpha e^{\beta t+x}
$$

where $\alpha, \beta$ only depend on $d, M_{0}, M_{1}$ and $\left\|u_{0}\right\|_{\left[L^{\infty}(\mathbb{R})\right]^{d}}$.

\section{Proof of Lemma 3.4:}

First we assume that $z$ is a smooth function. We will show that $z(t, x)$ becomes exponentially small on a domain of the form $\{\beta t+x<0\}$. Indeed, any solution of (3.7) admits the integral representation

$$
\begin{aligned}
z^{i}(t, x)=G(t) * z^{i}(0) & -\int_{0}^{t}\left(\partial_{x} G(t-s)\right) *\left[\lambda^{i}(u) z^{i}\right](s) d s \\
& +\int_{0}^{t} G(t-s) *\left[\sum_{j=1, \ldots, d} \lambda_{, j}^{i}(u)\left[z^{i} \partial_{x} u^{j}-z^{j} \partial_{x} u^{i}\right]\right]
\end{aligned}
$$

in terms of convolutions with standard heat kernel $G(t)=G(t, x)=\frac{1}{\sqrt{4 \pi t}} e^{\frac{-x^{2}}{4 t}}$. Therefore

$$
\begin{aligned}
|z(t, x)| \leq \int_{\mathbb{R}} G(t, x-y)|z(0, y)| d y & +M_{0} \int_{0}^{t} \int_{\mathbb{R}}\left|\left(\partial_{x} G(t-s, x-y)\right)\right||z(s, y)| d s d y \\
& +2 M_{1} \int_{0}^{t} \int_{\mathbb{R}} G(t-s, x-y)\left\|\partial_{x} u(s)\right\|_{\left[L^{\infty}(\mathbb{R})\right]^{d}}|z(s, y)| d s d y .
\end{aligned}
$$

We know that there exists a function $B$ satisfying $B(t) \leq 2 e^{C t}$ for every $t>0$, for some constant $C$ depending only on $M_{0}$, such that

$$
E(t, x)=B(t) \exp \left(4 M_{1} \int_{0}^{t}\left\|\partial_{x} u(s)\right\|_{\left[L^{\infty}(\mathbb{R})\right]^{d}} d s\right) e^{t+x},
$$

satisfies the following estimates (see Bianchini et al. [四] inequalities (12.8)-(12.9)-(12.10)):

$$
\left\{\begin{array}{l}
\int_{\mathbb{R}} G(t, x-y)|z(0, y)| d y<\frac{1}{\sqrt{4 \pi t}} \int_{\mathbb{R}} e^{\frac{-(x-y)^{2}}{4 t}} e^{y} d y=e^{t+x} \\
M_{0} \int_{0}^{t} \int_{\mathbb{R}} \mid\left(\partial_{x} G(t-s, x-y)\right) E(s, y) d s d y \leq \frac{1}{2} E(t, x)-\frac{1}{2} e^{t+x} \\
2 M_{1} \int_{0}^{t} \int_{\mathbb{R}} G(t-s, x-y)\left\|\partial_{x} u(s)\right\|_{\left[L^{\infty}(\mathbb{R})\right]^{d}} E(s, y) d s d y \leq \frac{1}{2} E(t, x)-\frac{1}{2} e^{t+x}
\end{array}\right.
$$

Notice that this result can also be checked directly by computation.

Thanks to the previous bounds and similarly as in the proof of (3.5), we obtain

$$
|z(t, x)| \leq E(t, x)
$$


Then using Sobolev injection, we deduce that

$$
|z(t, x)| \leq E(t, x) \leq 2 e^{C t} \exp \left(4 M_{1} \int_{0}^{t}\left\|\partial_{x x} u(s)\right\|_{\left[L^{1}(\mathbb{R})\right]^{d}} d s\right) e^{t+x} .
$$

Finally, using Corollary 3.3 (with $\varepsilon=1$ ), we deduce that

$$
|z(t, x)| \leq 2 e^{C t} \exp \left(8 d M_{1} C_{0}\left(2 \sqrt{t}+\frac{t}{\sqrt{T_{0}}}\right)\right) e^{t+x} .
$$

We observe that this estimate only depends on $d, M_{0}, M_{1}$ and $\left\|u_{0}\right\|_{\left[L^{\infty}(\mathbb{R})\right]^{d}}$. We can prove the same bound for general $z$, not necessarily smooth, using again an approximation argument joint to the continuity of the solution of (3.7) with respect to its initial data.

\subsection{Propagation speed}

Consider two solutions $u^{\varepsilon}, v^{\varepsilon}$ of the same viscous system (2.1), whose initial data coincide inside a bounded interval $[a, b]$. Since the system is parabolic, at a given time $t>0$ one may well have $u^{\varepsilon}(t, x) \neq v^{\varepsilon}(t, x)$ for all $x \in \mathbb{R}$. Yet, we want to show that the difference $\left|u^{\varepsilon}-v^{\varepsilon}\right|$ remains small once it is confined within a bounded interval $[a+\beta t, b-\beta t]$. This result will be useful in the Subsection 3.3, because it implies the uniqueness of the continuous vanishing viscosity solutions and of the semigroup.

\section{Lemma 3.5 (Propagation speed)}

For some constants $\alpha, \beta>0$ independent of $\varepsilon$, the following holds. Let $u^{\varepsilon}=\left(u^{\varepsilon, i}\right)_{i=1, \ldots, d}$ and $v^{\varepsilon}=\left(v^{\varepsilon, i}\right)_{i=1, \ldots, d}$ be the two solutions of system (2.1), given by Theorem 2.1 (i), whose initial data satisfy, for all reals $a<b$ :

$$
u^{\varepsilon}(0, x)=v^{\varepsilon}(0, x) \quad \text { for } \quad x \in[a, b] .
$$

Then for all $x \in \mathbb{R}, t>0$ one has

$$
\left|u^{\varepsilon}(t, x)-v^{\varepsilon}(t, x)\right| \leq \alpha\left\|u^{\varepsilon}(0, \cdot)-v^{\varepsilon}(0, \cdot)\right\|_{L^{\infty}(\mathbb{R})}\left(e^{\frac{\beta t-(x-a)}{\varepsilon}}+e^{\frac{\beta t+(x-b)}{\varepsilon}}\right) .
$$

Proof of Lemma 3.5: We prove this lemma in three Steps.

Step 1. As a first step we consider a solution $z$ of system (3.7) (for $\varepsilon=1$ ), whose initial data satisfies

$$
\begin{cases}|z(0, x)| \leq M & \text { if } \quad x \geq b \\ z(0, x)=0 & \text { if } \quad x<b .\end{cases}
$$

By the linearity of system (3.7) and "translation invariance", an application of Lemma 3.4 to the translated solution, yields

$$
|z(t, x)| \leq M \alpha e^{\beta t+(x-b)}
$$

On the other hand, if

$$
\begin{cases}|z(0, x)| \leq M & \text { if } \quad x \leq a \\ z(0, x)=0 & \text { if } \quad x>a\end{cases}
$$

then (using translation and the symmetry $x \mapsto-x$ ) 


$$
|z(t, x)| \leq M \alpha e^{\beta t-(x-a)} .
$$

Step 2. (Case $\varepsilon=1$ ): In this step we prove the result in the particular case $\varepsilon=1$. Let $u$ and $v$ be two solutions of system (2.1), with $\varepsilon=1$. We consider a third solution $w$ of (2.1) with initial data

$$
w(0, x)=\left\{\begin{array}{lll}
u(0, x) & \text { if } \quad x \leq b \\
v(0, x) & \text { if } \quad x \geq b
\end{array}\right.
$$

For $0<\theta<1$, we set

$$
u^{\theta}(t=0, x)=u_{0}^{\theta}(x)=\theta u(0, x)+(1-\theta) w(0, x)
$$

and we call $u^{\theta}=\left(u^{\theta, i}\right)_{i=1, \ldots, d}$ the solution given by Theorem 2.1 (i), of (2.1), with $\varepsilon=1$ and initial data $u_{0}^{\theta}$. Using system (2.1), we can check that the tangent vector

$$
\left(z^{\theta, i}\right)_{i=1, \ldots, d}=z^{\theta}=\frac{d u^{\theta}}{d \theta}
$$

is a solution of the following Cauchy problem:

$$
\begin{gathered}
\partial_{t} z^{\theta, i}+\partial_{x}\left(\lambda^{i}\left(u^{\theta}\right) z^{\theta, i}\right)+\partial_{x x} z^{\theta, i}=\sum_{j=1, \cdots, d} \lambda_{, j}^{i}\left(u^{\theta}\right)\left[z^{\theta, i} \partial_{x} u^{\theta, j}-z^{\theta, j} \partial_{x} u^{\theta, i}\right] \text { for } i=1, \cdots, d \\
z^{\theta}(0, x)=u(0, x)-w(0, x) .
\end{gathered}
$$

If (3.8) holds, then by previous analysis all functions $z^{\theta}$ satisfy the following inequality

$$
\left|z^{\theta}(t, x)\right| \leq \alpha\|u(0, \cdot)-v(0, \cdot)\|_{L^{\infty}(\mathbb{R})} e^{\beta t+(x-b)} .
$$

Therefore

$$
|u(t, x)-w(t, x)| \leq \int_{0}^{1}\left|z^{\theta}(t, x)\right| d \theta \leq \alpha\|u(0, \cdot)-v(0, \cdot)\|_{L^{\infty}(\mathbb{R})} e^{\beta t+(x-b)} .
$$

Similarly, we can prove that

$$
|v(t, x)-w(t, x)| \leq \alpha\|u(0, \cdot)-v(0, \cdot)\|_{L^{\infty}(\mathbb{R})} e^{\beta t-(x-a)} .
$$

Collecting (3.9) with (3.10), we get

$$
|u(t, x)-v(t, x)| \leq \alpha\|u(0, \cdot)-v(0, \cdot)\|_{L^{\infty}(\mathbb{R})}\left(e^{\beta t-(x-a)}+e^{\beta t+(x-b)}\right) .
$$

$\underline{\text { Step 3. (Case } \varepsilon>0)}$ : Rescaling in time and in space, with $u^{\varepsilon}(t, x)=u\left(\frac{t}{\varepsilon}, \frac{x}{\varepsilon}\right)$ and $v^{\varepsilon}(t, x)=$

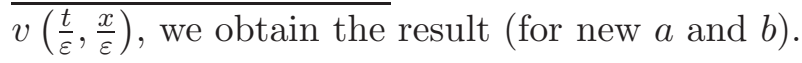




\subsection{Continuous vanishing viscosity solutions and $L^{1}$-stability estimate}

In this subsection we give the definition of continuous vanishing viscosity solutions and we prove that the solution of system (1.1)-(1.2), constructed in Theorem 1.1 (i), is the unique continuous vanishing viscosity solution (Theorem 1.1 (ii)). The proof of $L^{1}$-stability estimate (Theorem 1.1 (iii)) is done at the end of this subsection. The idea of the proof is the following: our solution is continuous with a control on the modulus of continuity. This implies that the total variation of the solution is locally small. Taking into account the finite propagation speed property, it is then possible to localize the argument developed in Bianchini et al. [4], and finally to extend it to the case of large initial data.

\section{Definition 3.6 (Continuous vanishing viscosity solutions)}

Let $T>0$. A function $u \in C((0,+\infty) \times \mathbb{R})$ is a viscosity solution of system (1.1) if for any small $\nu>0$ there exists a constant $\eta>0$ such that, for all $t \in[0, T]$ the function $u(t, \cdot)$ has a total variation smaller than $\nu$ on any interval $[a, b]$ where $b-a \leq \eta$ and moreover the following integral estimate hold.

There exist constants $C, \gamma>0$ (depending on $\eta$ ) such that, for every $\tau \geq 0$ and $a<\xi<b$, with $b-a \leq \eta$, one has

$$
\limsup _{h \rightarrow 0^{+}} \frac{1}{h} \int_{a+\gamma h}^{b-\gamma h}\left|u(\tau+h, x)-U_{(u ; \tau, \xi)}^{b}(h, x)\right| d x \leq C(T V[u(\tau) ;(a, b)])^{2}
$$

where $T V[u(\tau) ;(a, b)]$ is the total variation of $u(\tau, \cdot)$ on the interval $(a, b)$ and $U_{(u ; \tau, \xi)}^{b}$ is the solution of the linear hyperbolic Cauchy problem with constant coefficients:

$$
\partial_{t} w^{i}+\lambda^{i}(u(\tau, \xi)) \partial_{x} w^{i}=0, \quad \text { with } \quad w^{i}(0, x)=u^{i}(\tau, x) .
$$

Now, we prove that our solution constructed in Theorem 1.1 (i) is a continuous vanishing viscosity solutions in the sense of this definition.

Theorem 3.7 (Existence of continuous vanishing viscosity solutions)

The solution of system (1.1), given by Theorem 1.1 (i), is a continuous vanishing viscosity solutions, in the sense of Definition 3.6.

To prove this Theorem, we need to recall the following Lemma.

\section{Lemma 3.8 (Solution with small total variation)}

For all $\xi \in \mathbb{R}$, let $v^{\varepsilon}=\left(v^{\varepsilon, i}\right)_{i=1, \ldots, d}, w^{\varepsilon}=\left(w^{\varepsilon, i}\right)_{i=1, \ldots, d}$ be respectively the two solutions of the viscous systems

$$
\begin{gathered}
\partial_{t} v^{\varepsilon, i}+\lambda^{i}\left(v^{\varepsilon}\right) \partial_{x} v^{\varepsilon, i}=\varepsilon \partial_{x x} v^{\varepsilon, i}, \\
\partial_{t} w^{\varepsilon, i}+\lambda^{i}\left(w^{\varepsilon}(0, \xi)\right) \partial_{x} w^{\varepsilon, i}=\varepsilon \partial_{x x} w^{\varepsilon, i}
\end{gathered}
$$

with the same initial data $v^{\varepsilon}(0, x)=w^{\varepsilon}(0, x)=\bar{u}(x)$, where $\bar{u}$ is a function with total variation smaller than $\nu>0$. If $\nu$ is small enough, then for all $h>0$, there exists a positive constant $C$ independent of $\varepsilon$, such that

$$
\left\|v^{\varepsilon}(h, \cdot)-w^{\varepsilon}(h, \cdot)\right\|_{\left[L^{1}(\mathbb{R})\right]^{d}} \leq C h(T V[\bar{u}])^{2} .
$$


For the proof of this Lemma see Bianchini et al. [4, Lemma 15.2] (Necessity).

Proof of Theorem 3.7:

Because the solution $u$ given by Theorem 1.1 (i) has a modulus of continuity controlled by (1.5), we can choose a constant $\eta>0$ such that for $a<b$ with $b-a \leq \eta$, we have

$$
T V[u(\tau) ;(a, b)] \leq \delta
$$

(i.e. $u(\tau, \cdot)$ has small total variation on $(a, b))$. To prove estimate (3.11), first, we fix $\tau$ and $\xi \in(a, b)$ and we define the following truncate function

$$
\bar{u}^{\varepsilon}(\tau)(x)=\bar{u}^{\varepsilon}(\tau, x)= \begin{cases}u^{\varepsilon}(\tau, a) & \text { if } \quad x \leq a, \\ u^{\varepsilon}(\tau, x) & \text { if } \quad a<x<b, \\ u^{\varepsilon}(\tau, b) & \text { if } \quad b \leq x,\end{cases}
$$

where $u^{\varepsilon}$ is the solution of (2.1), constructed in Theorem 2.1 (i). Call $v^{\varepsilon}=\left(v^{\varepsilon, i}\right)_{i=1, \ldots, d}$, $w^{\varepsilon}=\left(w^{\varepsilon, i}\right)_{i=1, \ldots, d}$ respectively the solutions of (3.12) and (3.13) with the same initial data $v^{\varepsilon}(0, x)=w^{\varepsilon}(0, x)=\bar{u}^{\varepsilon}(\tau, x)$. Let $U_{(u ; \tau, \xi)}^{b, \varepsilon}=\left(U_{(u ; \tau, \xi)}^{b, \varepsilon, i}\right)_{i=1, \ldots, d}$ be the solution of the parabolic Cauchy problem (3.13) with $U_{(u ; \tau, \xi)}^{b, \varepsilon}(0, x)=u^{\varepsilon}(\tau, x)$. Let $\beta$ be the positive constant defined in Lemma 3.5. Then by definition of $u$ and $U_{(u ; \tau, \xi)}^{b}$, we can see that

$$
\frac{1}{h} \int_{a+\beta h}^{b-\beta h}\left|u(\tau+h, x)-U_{(u ; \tau, \xi)}^{b}(h, x)\right| d x \leq \lim _{\varepsilon \rightarrow 0} \frac{1}{h} \int_{a+\beta h}^{b-\beta h}\left|u^{\varepsilon}(\tau+h, x)-U_{(u ; \tau, \xi)}^{b, \varepsilon}(h, x)\right| d x .
$$

This implies that

$$
\begin{aligned}
\frac{1}{h} \int_{a+\beta h}^{b-\beta h}\left|u(\tau+h, x)-U_{(u ; \tau, \xi)}^{b}(h, x)\right| d x \leq & \leq \underbrace{\lim _{\varepsilon \rightarrow 0} \frac{1}{h} \int_{a+\beta h}^{b-\beta h}\left|u^{\varepsilon}(\tau+h, x)-v^{\varepsilon}(h, x)\right| d x}_{I_{1}^{\varepsilon}} \\
& +\underbrace{\lim _{\varepsilon \rightarrow 0} \frac{1}{h} \int_{a+\beta h}^{b-\beta h}\left|v^{\varepsilon}(h, x)-w^{\varepsilon}(h, x)\right| d x}_{I_{2}^{\varepsilon}} \\
& +\underbrace{\lim _{\varepsilon \rightarrow 0} \frac{1}{h} \int_{a+\beta h}^{b-\beta h}\left|w^{\varepsilon}(h, x)-U_{(u ; \tau, \xi)}^{b, \varepsilon}(h, x)\right| d x}_{I_{3}^{\varepsilon}} .
\end{aligned}
$$

Using Lemma 3.5 on the finite propagation speed and estimate (2.2), we obtain, for $h$ small enough, that $\lim _{\varepsilon \rightarrow 0} I_{1}^{\varepsilon}+I_{3}^{\varepsilon}=0$. Moreover, by Lemma 3.8, we know that,

$$
\limsup _{\varepsilon \rightarrow 0} I_{2}^{\varepsilon} \leq \limsup _{\varepsilon \rightarrow 0} C\left(T V\left[\bar{u}^{\varepsilon}(\tau)\right]\right)^{2} \leq C(T V[u(\tau) ;(a, b)])^{2}
$$


which ends the proof of Theorem 3.7 .

Before going into the proof of Theorem 1.1 (ii) and (iii), we first recall in Lemma 3.9 the continuous $L^{1}$ estimate, proved by Bianchini et al. in [4]. Then we prove Proposition 3.10 that claims that our solution coincides locally with the semigroup vanishing viscosity solutions defined by Bianchini et al. in [河. Let us underline that the semigroup of Bianchini-Bressan is defined for initial data which are not necessarily continuous, but with small total variation.

\section{Lemma 3.9 ( $L^{1}$ estimate for initial data with small total variation)}

Let $S_{t}$ be the semigroup of vanishing viscosity solutions, constructed by Bianchini et al. in [4] as the limit in $L_{\text {loc }}^{1}(\mathbb{R})$ of a sequence $S^{\varepsilon}$ (see [4, (13.9)]). Consider any interval $[a, b]$ and two initial data $\bar{u}, \bar{v} \in L_{l o c}^{1}(\mathbb{R})$ with small total variation. Then, the following continuous $L^{1}$ estimate holds.

$$
\begin{gathered}
\int_{a+\beta t}^{b-\beta t}\left|\left(S_{t} \bar{u}\right)(x)-\left(S_{t} \bar{v}\right)(x)\right| d x \leq L_{0} \int_{a}^{b}|\bar{u}(x)-\bar{v}(x)| d x \quad \text { for all } \quad 0 \leq t \leq \frac{b-a}{4 \beta}, \\
\left\|\left(S_{t}^{\varepsilon} \bar{u}\right)(x)-\left(S_{t}^{\varepsilon} \bar{v}\right)(x)\right\|_{\left[L^{1}(\mathbb{R})\right]^{d}} \leq L_{0}\|\bar{u}(x)-\bar{v}(x)\|_{\left[L^{1}(\mathbb{R})\right]^{d}} \quad \text { for all } \quad t \geq 0,
\end{gathered}
$$

where $\beta$ is the constant defined in Lemma 3.5 and $L_{0}$ is a positive constant independent of $\varepsilon$. For the proof of this lemma see Bianchini et al. 四, (13.13), (13.5)].

Now we prove the following proposition, which shows that our solution is locally a semigroup.

Proposition 3.10 (Semigroup for continuous vanishing viscosity solutions)

Let $u$ be a solution of system (1.1), given by Theorem 1.1 (i). Then, for all $T>0$, there exists $\eta>0$ only depending on $T, d, M_{0}, M_{1}, \Lambda$ and bounds on $\left\|u_{0}\right\|_{\left[L^{\infty}(\mathbb{R})\right]^{d}}$ and $\left\|\partial_{x} u_{0}\right\|_{[L \log L(\mathbb{R})]^{d}}$, such that for all $0<b-a \leq \eta$ and $\tau \in[0, T]$, we have

$$
\int_{a+\beta t}^{b-\beta t}\left|u(\tau+t, x)-\left(S_{t} \bar{u}(\tau)\right)(x)\right| d x=0, \quad \text { for all } \quad 0 \leq t \leq \frac{b-a}{4 \beta},
$$

where $\beta$ is the constant defined in Lemma 3.5, $S_{t}$ is the semigroup of vanishing viscosity solution defined by Bianchini et al. in [4] and $\bar{u}$ is the following truncate function

$$
\bar{u}(\tau)(x)= \begin{cases}u(\tau, a) & \text { if } x \leq a, \\ u(\tau, x) & \text { if } a<x<b, \\ u(\tau, b) & \text { if } b \leq x .\end{cases}
$$

\section{Proof of Proposition 3.10:}

First we remark that the solution $u$, given by Theorem 1.1 (i) satisfies (1.5), and then we can choose a constant $\eta>0$ such that for all $b-a \leq \eta$ the function $\bar{u}(\tau)$ has small total variation on $(a, b)$. Then, adopting the semigroup notation, we can write the vanishing viscosity solution defined by Bianchini et al. in [4] as $S_{t}(\bar{u}(\tau))$. The fact that $S_{t}$ is a semigroup is a consequence of the theory of Bianchini-Bressan developed in [4]. By construction of the solutions, we can write 


$$
\int_{a+\beta t}^{b-\beta t}\left|u(\tau+t, x)-S_{t}(\bar{u}(\tau))(x)\right| d x \leq \lim _{\varepsilon \rightarrow 0} \int_{a+\beta t}^{b-\beta t}\left|u^{\varepsilon}(\tau+t, x)-S_{t}^{\varepsilon}(\bar{u}(\tau))(x)\right| d x
$$

where $u^{\varepsilon}$ is the solution of (2.1), constructed in Theorem 2.1 (i). Here $S_{t}^{\varepsilon}(\bar{u}(\tau))$ is the semigroup solution of (3.12) with initial data $\bar{u}$, constructed by Bianchini-Bressan in 4 . Now, we add and we subtract in (3.18) the function $S_{t}^{\varepsilon}\left(\bar{u}^{\varepsilon}(\tau)\right.$ ), where $\bar{u}^{\varepsilon}(\tau)$ is the truncate function of $u^{\varepsilon}$ defined in (3.14), we deduce that, there exists two positive constants $C$ and $L_{0}$ independent of $\varepsilon$ such that

$$
\begin{aligned}
\int_{a+\beta t}^{b-\beta t}\left|u(\tau+t, x)-S_{t}(\bar{u}(\tau))(x)\right| d x \leq & \lim _{\varepsilon \rightarrow 0} \int_{a+\beta t}^{b-\beta t}\left|u^{\varepsilon}(\tau+t, x)-S_{t}^{\varepsilon}\left(\bar{u}^{\varepsilon}(\tau)\right)(x)\right| d x \\
& +\lim _{\varepsilon \rightarrow 0} \int_{a+\beta t}^{b-\beta t}\left|S_{t}^{\varepsilon}\left(\bar{u}^{\varepsilon}(\tau)\right)(x)-S_{t}^{\varepsilon}(\bar{u}(\tau))(x)\right| d x \\
\leq & \lim _{\varepsilon \rightarrow 0} C \int_{a+\beta t}^{b-\beta t}\left(e^{\frac{\beta t-(x-a)}{\varepsilon}}+e^{\frac{\beta t+(x-b)}{\varepsilon}}\right) d x \\
& +\lim _{\varepsilon \rightarrow 0} L_{0}\left\|\bar{u}(\tau)-\bar{u}^{\varepsilon}(\tau)\right\|_{\left[L^{1}(\mathbb{R})\right]^{d}}
\end{aligned}
$$

where we have used in the second inequality the finite propagation speed Lemma 3.5 with estimate (2.2) and estimate (3.16). Using the fact that $u^{\varepsilon}$ converges, as $\varepsilon \rightarrow 0$, to $u$ in $L_{l o c}^{\infty}([0,+\infty) \times \mathbb{R})$ and

$$
\lim _{\varepsilon \rightarrow 0} C\left(e^{\frac{\beta t-(x-a)}{\varepsilon}}+e^{\frac{\beta t+(x-b)}{\varepsilon}}\right)=0 \quad \text { on } \quad[a+\beta t, b-\beta t]
$$

we obtain the result.

Now, we prove that the solution $u$ constructed in Theorem 1.1 (i), is the unique continuous vanishing viscosity solution of system (1.1)-(1.2), in the sense of Definition 3.6.

Proof of Theorem 1.1 (ii):

Step 1. (Short time): Let $w=w(t, x)$ be a continuous vanishing viscosity solution of (1.1) and $u$ be a solution of (1.1) constructed in Theorem 1.1 (i). Assume $w(0, x)=u(0, x)$. By Definition 3.6, we know that there exists two constants $\gamma$ and $\eta$ such that $w$ satisfies (3.11). Let us call $\left(\eta_{0}, \beta\right)$ the parameters given by Proposition 3.10. Then up to decreasing $\eta_{0}$ and increasing $\beta$, we can assume that $\eta_{0}=\eta$ and $\beta=\gamma$. Given any interval $[a, b]$, such that $b-a=\eta$, thanks to identity (3.17) (with $\tau=0$ ) and $w(0, x)=u(0, x)$ we have

$$
\int_{a+t \beta}^{b-t \beta}|w(t, x)-u(t, x)| d x=\int_{a+t \beta}^{b-t \beta}\left|\bar{w}(t, x)-\left(S_{t} \bar{w}(0)\right)(x)\right| d x, \quad \text { for all } \quad t \leq \frac{\eta}{4 \beta}
$$

where 


$$
\bar{w}(t)(x)=\bar{w}(t, x)= \begin{cases}w(t, a) & \text { if } \quad x \leq a, \\ w(t, x) & \text { if } a<x<b, \\ w(t, b) & \text { if } \quad b \leq x .\end{cases}
$$

Let $L_{0}$ be the Lipschitz constant of the semigroup $S_{t}$, defined in (3.15). Using estimate (3.15) (and the fact that $S_{t}(\bar{w}(0))$ is continuous in $t$ with values in $L^{1}(\mathbb{R})$ ), we get the following error estimate

$$
\begin{aligned}
\int_{a+t \beta}^{b-t \beta} & \left|\bar{w}(t, x)-\left(S_{t} \bar{w}(0)\right)(x)\right| d x \\
& \leq L_{0} \int_{0}^{t}\left[\limsup _{h \rightarrow 0^{+}} \frac{1}{h} \int_{a+(\tau+h) \beta}^{b-(\tau+h) \beta}\left|\bar{w}(\tau+h, x)-S_{h}(\bar{w}(\tau))(x)\right| d x\right] d \tau
\end{aligned}
$$

as in Bianchini et al. [4, (15.9)]. Now, to prove the uniqueness it thus suffices to show that the integrand on the right hand side of (3.19) vanishes for $\tau \in[0, t]$. Fix any $\tau \in[0, t]$ and let $\varepsilon>0$ be given. We can choose finitely many points

$$
a+\tau \beta=x_{0}<x_{1}<\cdots<x_{N}=b-\tau \beta,
$$

such that, for every $j=1, \ldots, N$,

$$
T V\left[\bar{w}(\tau, \cdot) ;\left(x_{j-1}, x_{j}\right)\right]<\varepsilon .
$$

By Theorem 3.7 and Proposition 3.10, the function $t \mapsto S_{t-\tau} \bar{w}(\tau)$ is itself a continuous vanishing viscosity solution and hence it also satisfies estimate (3.11). We now consider the mid point $y_{j}=\frac{x_{j-1}+x_{j}}{2}$. Using the estimate (3.11) with $\xi=y_{j}$ on each interval $\left(x_{j-1}, x_{j}\right)$, we compute

$$
\begin{aligned}
\limsup _{h \rightarrow 0^{+}} & \frac{1}{h} \int_{a+(\tau+h) \beta}^{b-(\tau+h) \beta}\left|\bar{w}(\tau+h, x)-S_{h}(\bar{w}(\tau))(x)\right| d x \\
\leq & \sum_{j=1}^{N} \limsup _{h \rightarrow 0^{+}} \frac{1}{h} \int_{x_{j-1}+h \beta}^{x_{j}-h \beta}\left|\bar{w}(\tau+h, x)-U_{\left(\bar{w} ; \tau, y_{j}\right)}^{b}(h, x)\right| d x \\
& +\sum_{j=1}^{N} \limsup _{h \rightarrow 0^{+}} \frac{1}{h} \int_{x_{j-1}+h \beta}^{x_{j}-h \beta}\left|U_{\left(\bar{w} ; \tau, y_{j}\right)}^{b}(h, x)-S_{h}(\bar{w}(\tau))(x)\right| d x .
\end{aligned}
$$

Using (3.20), we obtain

$$
\begin{aligned}
\limsup _{h \rightarrow 0^{+}} \frac{1}{h} \int_{a+(\tau+h) \beta}^{b-(\tau+h) \beta}\left|\bar{w}(\tau+h, x)-S_{h}(\bar{w}(\tau))(x)\right| d x & \leq C_{1} \sum_{j=1}^{N}\left(T V\left[\bar{w}(\tau, \cdot) ;\left(x_{j-1}, x_{j}\right)\right]\right)^{2} \\
& \leq C_{1} \varepsilon T V[\bar{w}(\tau, \cdot) ;(a+\tau \beta, b-\tau \beta)] \\
& \leq C_{1} \varepsilon,
\end{aligned}
$$


because $T V[\bar{w}(\tau, \cdot) ;(a+\tau \beta, b-\tau \beta)] \leq \nu \leq 1$ (for a suitable choice of $\nu$ in Definition 3.6).

Since $\varepsilon>0$ was arbitrary, the integrand on the right hand side of (3.19) must vanish at time $\tau \in[0, t]$, with $t \leq \frac{\eta}{4 \beta}$.

Step 2. (Long time): Since the constants $L_{0}$ and $C_{1}$ are uniform on $(0, T)$, for all $T>0$ we can find $n_{0} \in \mathbb{N}$, such that $\frac{n_{0}+2}{2} \frac{\eta}{4 \beta} \geq T$. We repeat the same argument, for all $n=1, \ldots, n_{0}$ on the interval $\left[\frac{n}{2} \frac{\eta}{4 \beta}, \frac{n+2}{2} \frac{\eta}{4 \beta}\right]$, we prove the uniqueness for all $t \in[0, T]$. This completes the proof.

In the following we prove the $L^{1}$-stability estimate announced in Theorem 1.1 (iii).

Proof of Theorem 1.1 (iii):

Step 1. (Local estimate): From Proposition 3.10, we know that, there exist two positive constants $\beta$ and $\eta$ depending only on $T, M_{0}, M_{1}, d, \Lambda$ and bounds on $\left\|u_{0}\right\|_{\left[L^{\infty}(\mathbb{R})\right]^{d}}$, $\left\|\partial_{x} u_{0}\right\|_{[L \log L(\mathbb{R})]^{d}},\left\|v_{0}\right\|_{\left[L^{\infty}(\mathbb{R})\right]^{d}},\left\|\partial_{x} v_{0}\right\|_{[L \log L(\mathbb{R})]^{d}}$, such that, for all $0<t \leq \frac{\eta}{4 \beta}$, we have

$$
\int_{a+\beta t}^{a+\eta-\beta t}|u(t, x)-v(t, x)| d x=\int_{a+\beta t}^{a+\eta-\beta t}\left|S_{t} \bar{u}_{0}(x)-S_{t} \bar{v}_{0}(x)\right| d x
$$

where $\bar{u}_{0}$ and $\bar{v}_{0}$ are the following truncate functions

$\bar{u}_{0}(x)=\left\{\begin{array}{ll}u_{0}(a) & \text { if } \quad x \leq a, \\ u_{0}(x) & \text { if } a<x<a+\eta, \\ u_{0}(a+\eta) & \text { if } a+\eta \leq x\end{array} \quad\right.$ and $\quad \bar{v}_{0}(x)= \begin{cases}v_{0}(a) & \text { if } \quad x \leq a, \\ v_{0}(x) & \text { if } a<x<a+\eta, \\ v_{0}(a+\eta) & \text { if } a+\eta \leq x .\end{cases}$

Using the continuous $L^{1}$ estimate (3.15), we get

$$
\int_{a+\beta t}^{a+\eta-\beta t}|u(t, x)-v(t, x)| d x \leq L_{0} \int_{a}^{a+\eta}\left|\bar{u}_{0}(x)-\bar{v}_{0}(x)\right| d x=L_{0} \int_{a}^{a+\eta}\left|u_{0}(x)-v_{0}(x)\right| d x .
$$

This leads to the following local estimate:

$$
\|u(h, \cdot)-v(h, \cdot)\|_{\left[L^{1}\left(I_{\eta}^{t}\right)\right]^{d}} \leq L_{0}\left\|u_{0}-v_{0}\right\|_{\left[L^{1}\left(I_{\eta}^{0}\right)\right]^{d}} \quad \text { for all } \quad 0<t \leq \frac{\eta}{4 \beta},
$$

where $I_{\eta}^{t}=[a+\beta t, a+\eta-\beta t]$.

Step 2. (Global estimate): For all $k \in \mathbb{Z}$, we note $I_{k}=\left[\frac{k}{2} \eta, \frac{k+2}{2} \eta\right]$ and $J_{k}=\left[\frac{2 k+1}{4} \eta, \frac{2 k+3}{4} \eta\right]$. We apply the local estimate (3.21), we obtain

$$
\|u(t, \cdot)-v(t, \cdot)\|_{\left[L^{1}\left(J_{k}\right)\right]^{d}} \leq L_{0}\left\|u_{0}-v_{0}\right\|_{\left[L^{1}\left(I_{k}\right)\right]^{d}} \quad \text { for all } \quad 0<t \leq \frac{\eta}{4 \beta} .
$$

Taking the sum over $k \in \mathbb{Z}$, we deduce that

$$
\|u(t, \cdot)-v(t, \cdot)\|_{\left[L^{1}(\mathbb{R})\right]^{d}} \leq 2 L_{0}\left\|u_{0}-v_{0}\right\|_{\left[L^{1}(\mathbb{R})\right]^{d}} \quad \text { for all } \quad 0<t \leq \frac{\eta}{4 \beta} .
$$


Now for all $T>0$, we know that there exists $n_{0} \in \mathbb{N}$, where $\frac{n_{0}+2}{2} \frac{\eta}{4 \beta} \geq T$. We repeat the previous estimate, for all $n=1, \ldots, n_{0}$ on the interval $\left[\frac{n}{2} \frac{\eta}{4 \beta}, \frac{n+2}{2} \frac{\eta}{4 \beta}\right]$, we obtain that there exists $L=L\left(\eta, \beta, n_{0}\right)$ such that

$$
\|u(t, \cdot)-v(t, \cdot)\|_{\left[L^{1}(\mathbb{R})\right]^{d}} \leq L\left\|u_{0}-v_{0}\right\|_{\left[L^{1}(\mathbb{R})\right]^{d}} \quad \text { for all } \quad 0<t \leq T
$$

which proves the result.

\section{Existence and uniqueness of Lipschitz solution}

This section is devoted to the proof of Theorem 1.3. We study Lipschitz solutions of system (1.1)-(1.2) and we show some uniqueness results for some particular matrices $\left(\lambda_{, j}^{i}(u)\right)_{i, j=1, \ldots, d}$ with $d \geq 2$. In the following subsection, we first recall the definition of viscosity solutions (different from Definition 3.6 for continuous vanishing viscosity solutions) and some well-known results in this framework. The proof of Theorem 1.3 is done in Subsection 4.2 .

\subsection{Some useful results for viscosity solutions}

The notion of viscosity solutions has been introduced by Crandall and Lions [6] in 1980, to solve first-order Hamilton-Jacobi equations. Let us mention that this theory has also been extended to the second order equations (see for instance the work of Jensen [16] and Ishii [13]). For a good introduction to this theory, we refer the reader for instance to Barles [3] and Bardi, Capuzzo-Dolcetta [2].

Now, we recall the definition of the viscosity solution for the following problem for all $0 \leq \varepsilon \leq 1$ satisfied by a real function $v(t, x)$ :

$$
\partial_{t} v+H\left(t, x, v, \partial_{x} v\right)-\varepsilon \partial_{x x} v=0 \text { for } x \in \mathbb{R}, t \in(0,+\infty) .
$$

where $H:(0,+\infty) \times \mathbb{R}^{3} \longmapsto \mathbb{R}$ is the Hamiltonian and is supposed to be continuous. We introduce the following set of functions, for a set $\Omega \subset \mathbb{R}^{N}$ :

$$
\begin{aligned}
U S C(\Omega) & =\{f: \Omega \longmapsto \mathbb{R} \text {, with } f \text { upper semicontinuous }\}, \\
L S C(\Omega) & =\{f: \Omega \longmapsto \mathbb{R} \text {, with } f \text { lower semicontinuous }\} .
\end{aligned}
$$

\section{Definition 4.1 (Viscosity subsolution, supersolution and solution)}

A function $v \in U S C((0,+\infty) \times \mathbb{R})$ is a viscosity subsolution of $(4.1)$ if for every $\left(t_{0}, x_{0}\right) \in$ $(0,+\infty) \times \mathbb{R}$ and for every test function $\phi \in C^{2}((0,+\infty) \times \mathbb{R})$, that is tangent from above to $v$ at $\left(t_{0}, x_{0}\right)$, the following holds:

$$
\partial_{t} \phi\left(t_{0}, x_{0}\right)+H\left(t_{0}, x_{0}, v\left(t_{0}, x_{0}\right), \partial_{x} \phi\left(t_{0}, x_{0}\right)\right)-\varepsilon \partial_{x x} \phi\left(t_{0}, x_{0}\right) \leq 0 .
$$

A function $v \in \operatorname{LSC}((0,+\infty) \times \mathbb{R})$ is a viscosity supersolution of (4.1) if for every $\left(t_{0}, x_{0}\right) \in$ $(0, T) \times \mathbb{R}$ and for every test function $\phi \in C^{2}((0,+\infty) \times \mathbb{R})$, that is tangent from below to $v$ at $\left(t_{0}, x_{0}\right)$, the following holds:

$$
\partial_{t} \phi\left(t_{0}, x_{0}\right)+H\left(t_{0}, x_{0}, v\left(t_{0}, x_{0}\right), \partial_{x} \phi\left(t_{0}, x_{0}\right)\right)-\varepsilon \partial_{x x} \phi\left(t_{0}, x_{0}\right) \geq 0 .
$$


A continuous function $v$ is a viscosity solution of (4.1) if, and only if, it is a sub and a supersolution of (4.1).

Remark 4.2 When $v$ is a subsolution (resp. supersolution) of (4.1), we write

$$
\partial_{t} v+H\left(t, x, v, \partial_{x} v\right)-\varepsilon \partial_{x x} v \leq 0 \quad\left(r e s p . \quad \partial_{t} v+H\left(t, x, v, \partial_{x} v\right)-\varepsilon \partial_{x x} v \geq 0\right) .
$$

Let us now recall some well-known results.

\section{Remark 4.3 (Classical solution-viscosity solution)}

If $v$ is a $C^{2}$ solution of (4.1), then $v$ is a viscosity solution of (4.1).

We now consider solutions of the following $O D E$ for $\alpha \in \mathbb{R}$ :

$$
\frac{d v}{d t}=\alpha v \quad \text { on } \quad(0,+\infty)
$$

A function $v:(0,+\infty) \longmapsto \mathbb{R}$ is said to be viscosity subsolution (resp. supersolution) if $v(t, x)=v(t)$ is a viscosity subsolution (resp. supersolution) of (4.1) with $H=-\alpha v, \varepsilon=0$ in the sense of Definition 4.1 .

\section{Lemma 4.4 (Gronwall lemma for viscosity solution)}

Let us consider a function $v \in U S C[0,+\infty)$, which is a viscosity subsolution of (4.9). Assume that $v(0) \leq v_{0}$ then $v(t) \leq v_{0} e^{\alpha t}$ for all $t \geq 0$.

The proof of this Lemma is a direct application of the comparison principle, (see Barles [3, Th $2.4])$.

\subsection{Uniqueness results for $W^{1, \infty}$ solutions}

In this subsection we prove Theorem 1.3. Before going on, we recall below in Theorem 4.5 a well-known uniqueness result for $W^{1, \infty}$ solutions of (1.1).

Theorem 4.5 (Existence and uniqueness of $W^{1, \infty}$ solution)

Assume $(H 1)$. Let $u_{0} \in\left[W^{1, \infty}(\mathbb{R})\right]^{d}$, such that $\partial_{x} u_{0} \geq 0$.

i) Then, for any $0<\varepsilon \leq 1$, there exists a function $u^{\varepsilon} \in \bigcap_{T>0}\left(\left[W^{2, \infty}([0, T) \times \mathbb{R})\right]^{d} \cap\left[C^{\infty}([0, T) \times \mathbb{R})\right]^{d}\right)$ solution of (2.1), such that for every fixed $t \in[0,+\infty)$ the function $u^{\varepsilon}(t, \cdot)$ is nondecreasing.

ii) If the solution $u^{\varepsilon}$ of (2.1) satisfies (for all $T>0$ )

$$
\left\|u^{\varepsilon}(t, \cdot)\right\|_{\left[W^{1, \infty}(\mathbb{R})\right]^{d}} \leq C_{T} \text { for all } t \in[0, T]
$$

with $C_{T}$ independent on $\varepsilon$, then $u^{\varepsilon}$ converges locally uniformly, as $\varepsilon \rightarrow 0$, to a function $u$ with $u \in \bigcap_{T>0}\left[W^{1, \infty}([0, T) \times \mathbb{R})\right]^{d}$. Moreover, this function $u$ is the unique solution of system (1.1) in the sense of distributions, assuming the solutions in $\bigcap_{T>0}\left[W^{1, \infty}([0, T) \times \mathbb{R})\right]^{d}$. 
The lines of the proof of this theorem are very standard (see for instance Cannone et al. [0] for a similar problem). For this reason, we skip the details of the proof, and notice that Theorem 4.5 follows from Remark 1.2 (i) and Theorem 2.1.

\section{Proof of Theorem 1.3:}

Using Theorem 4.5, it is enough to show that system (1.1)-(1.2) admits a solution satisfying (4.3). Indeed, we then get the same property for $\partial_{x} u$, where $u$ is the limit of $u^{\varepsilon}$ as $\varepsilon \rightarrow 0$. Moreover, from system (1.1) satisfied by $u$ and the fact that

$$
u \in\left[L^{\infty}((0,+\infty) \times \mathbb{R})\right]^{d} \quad \text { and } \quad \partial_{x} u \in\left[L^{\infty}((0,+\infty) \times \mathbb{R})\right]^{d},
$$

we deduce that $\partial_{t} u \in\left[L^{\infty}((0,+\infty) \times \mathbb{R})\right]^{d}$ which shows that $u \in\left[W^{1, \infty}([0,+\infty) \times \mathbb{R})\right]^{d}$.

To simplify, we set $w^{\varepsilon}=\partial_{x} u^{\varepsilon}$. Moreover, by Theorem 4.5 (i), we know that, $w^{\varepsilon, i} \geq 0$ and there exists a positive constant $C_{T}^{\varepsilon}$, such that for all $(t, x) \in(0, T) \times \mathbb{R}$ and for $i=1, \ldots, d$

$$
\left|u^{\varepsilon, i}\right|+\left|\partial_{x} u^{\varepsilon, i}\right|+\left|\partial_{t} u^{\varepsilon, i}\right|+\left|\partial_{t x} u^{\varepsilon, i}\right|+\left|\partial_{x x} u^{\varepsilon, i}\right|+\left|\partial_{t t} u^{\varepsilon, i}\right| \leq C_{T}^{\varepsilon},
$$

which implies in particular that

$$
\int_{\mathbb{R}}\left|w^{\varepsilon, i}(t, x)\right| d x \leq 2 C_{T}^{\varepsilon}
$$

We are interested in the quantity

$$
m_{i}(t)=\sup _{x \in \mathbb{R}} w^{\varepsilon, i}(t, x) .
$$

which also satisfies $\left|\partial_{t} m_{i}\right| \leq C_{T}^{\varepsilon}$. This supremum is reached at least at some point $x_{i}(t)$, because $w^{\varepsilon, i} \in W^{1, \infty}([0, T) \times \mathbb{R}) \cap L^{1}([0, T) \times \mathbb{R})$ and then for each $t \in[0, T), w^{\varepsilon, i}(t, x) \rightarrow 0$ as $|x| \rightarrow+\infty$.

In the following we prove that $m_{i}$ is bounded uniformly in $\varepsilon$ for all $i=1, \ldots, d$ which will imply the first point of the Theorem.

First, taking the derivative with respect to $x$, equation (2.1) satisfied by $u^{\varepsilon} \in$ $\left[C^{\infty}((0, T) \times \mathbb{R})\right]^{d}$, we can see that $w^{\varepsilon}$ satisfies the following equation

$$
\partial_{t} w^{\varepsilon, i}+\lambda^{i}\left(u^{\varepsilon}\right) \partial_{x} w^{\varepsilon, i}+\sum_{j=1, \ldots, d} \lambda_{, j}^{i}\left(u^{\varepsilon}\right) w^{\varepsilon, j} w^{\varepsilon, i}=\varepsilon \partial_{x x} w^{\varepsilon, i} .
$$

Now, we prove that $m_{i}$ is a viscosity subsolution of the following equation

$$
\frac{d}{d t} m_{i}(t)+\sum_{j=1, \ldots, d} \lambda_{, j}^{i}\left(u^{\varepsilon}\left(t, x_{i}(t)\right)\right) w^{\varepsilon, j}\left(t, x_{i}(t)\right) w^{\varepsilon, i}\left(t, x_{i}(t)\right)=0 .
$$

Indeed, let $\phi \in C^{2}(0, T)$ be a test function, such that $\phi \geq m_{i}$ and $\phi\left(t_{0}\right)=m_{i}\left(t_{0}\right)$ for some $t_{0} \in(0, T)$. From the definition of $m_{i}$, we can easily check that $\phi(t) \geq w^{\varepsilon, i}(t, x)$ for all $(t, x) \in[0, T) \times \mathbb{R}$ and $\phi\left(t_{0}\right)=w^{\varepsilon, i}\left(t_{0}, x_{i}\left(t_{0}\right)\right)$. From the fact that $w^{\varepsilon, i} \in C^{\infty}((0, T) \times \mathbb{R})$, by 
Remark 4.3 we know that $w^{\varepsilon, i}$ is a viscosity solution of (4.5). We apply Definition 4.1, and using the fact that $\partial_{x} \phi=\partial_{x x} \phi=0$, we get

$$
\frac{d}{d t} \phi\left(t_{0}\right)+\sum_{j=1, \ldots, d} \lambda_{, j}^{i}\left(u^{\varepsilon}\left(t_{0}, x_{i}\left(t_{0}\right)\right)\right) w^{\varepsilon, j}\left(t_{0}, x_{i}\left(t_{0}\right)\right) w^{\varepsilon, i}\left(t_{0}, x_{i}\left(t_{0}\right)\right) \leq 0 .
$$

This proves that $m_{i}$ is a viscosity subsolution of (4.6).

Notice that in the case $d=1$ each assumption $(K 1),(K 2)$ or $(K 3)$ reduces to $\lambda_{, 1}^{1}(u) \geq 0$ which corresponds to the well-known case of scalar Burgers equation with non shocks when the initial data is non-decreasing. For this reason in the following we consider the case $d \geq 2$. We will establish estimates on $m_{i}$ at the level $\varepsilon$, and the result for $\varepsilon=0$ is then a straightforward consequence passing to the limit in $\varepsilon$. Three cases may occur:

1- The case where $(K 1)$ holds: We see that $m_{1}$, satisfies (in the viscosity sense)

$$
\frac{d}{d t} m_{1}(t) \leq-\sum_{j=1, \ldots, d} \lambda_{, j}^{1}\left(u^{\varepsilon}\left(t, x_{1}(t)\right) w^{\varepsilon, j}\left(t, x_{1}(t)\right) w^{\varepsilon, 1}\left(t, x_{1}(t)\right) \leq 0,\right.
$$

where we have used the fact that, for $j=1, \ldots, d, \lambda_{, j}^{1}\left(u^{\varepsilon}\right) \geq 0$ and $w^{\varepsilon, j} \geq 0$. This proves by Lemma 4.4 (with $\alpha=0$ ) that,

$$
m_{1}(t) \leq m_{1}(0)=w^{\varepsilon, 1}\left(0, x_{1}(0)\right) \leq\left\|\partial_{x} u_{0}^{1, \varepsilon}\right\|_{L^{\infty}(\mathbb{R})} \leq\left\|\partial_{x} u_{0}^{1}\right\|_{L^{\infty}(\mathbb{R})}=: C_{1}(t) .
$$

By recurrence, we assume that $m_{j}(t) \leq C_{i}(t)$ for all $j \leq i$, where $C_{i}$ is a positive function independent of $\varepsilon$, and we prove that $m_{i+1}$ is bounded uniformly in $\varepsilon$. Indeed, we know that

$$
\begin{aligned}
\frac{d}{d t} m_{i+1}(t) \leq & \left.-\sum_{j=1, \ldots, d} \lambda_{, j}^{i+1}\left(u^{\varepsilon}\left(t, x_{i+1}(t)\right)\right)\right) w^{\varepsilon, j}\left(t, x_{i+1}(t)\right) w^{\varepsilon, i+1}\left(t, x_{i+1}(t)\right), \\
\leq & -\sum_{j \leq i} \lambda_{, j}^{i+1}\left(u^{\varepsilon}\left(t, x_{i+1}(t)\right)\right) w^{\varepsilon, j}\left(t, x_{i+1}(t)\right) w^{\varepsilon, i+1}\left(t, x_{i+1}(t)\right) \\
& -\sum_{i+1 \leq j \leq d} \lambda_{, j}^{i+1}\left(u^{\varepsilon}\left(t, x_{i+1}(t)\right)\right) w^{\varepsilon, j}\left(t, x_{i+1}(t)\right) w^{\varepsilon, i+1}\left(t, x_{i+1}(t)\right) .
\end{aligned}
$$

We use that $\lambda_{, j}^{i+1}\left(u^{\varepsilon}\right) \geq 0$, for $i+1 \leq j \leq d$ and we obtain that

$$
\begin{aligned}
\frac{d}{d t} m_{i+1}(t) & \leq-\sum_{j \leq i} \lambda_{, j}^{i+1}\left(u^{\varepsilon}\left(t, x_{i+1}(t)\right)\right) w^{\varepsilon, j}\left(t, x_{i+1}(t)\right) w^{\varepsilon, i+1}\left(t, x_{i+1}(t)\right) \\
& \leq d M_{1} m_{i+1}(t) C_{i}(t) .
\end{aligned}
$$

where we have used the assumption $m_{j} \leq C_{i}$ for all $j \leq i$. This implies by Lemma 4.4, with $\alpha=d M_{1}$, that

$$
\begin{aligned}
m_{i+1}(t) & \leq m_{i+1}(0) e^{\alpha \int_{0}^{t} C_{i}(s) d s}, \\
& \leq\left\|\partial_{x} u_{0}^{i+1}\right\|_{L^{\infty}(\mathbb{R})} e^{\alpha \int_{0}^{t} C_{i}(s) d s}:=C_{i+1}(t) .
\end{aligned}
$$


This proves that, for all $i=1, \ldots, d, m_{i}$ is bounded uniformly in $\varepsilon$, on each time interval $[0, T]$.

2- The case where $(K 2)$ holds: From (4.6), we obtain that,

$$
\begin{aligned}
\frac{d}{d t} m_{i}(t) & \leq-\sum_{j=1, \ldots, d} \lambda_{, j}^{i}\left(u^{\varepsilon}\left(t, x_{i}(t)\right)\right) w^{\varepsilon, j}\left(t, x_{i}(t)\right) w^{\varepsilon, i}\left(t, x_{i}(t)\right) \\
& \leq-\sum_{j=1, \ldots, d} A_{i j} w^{\varepsilon, j}\left(t, x_{i}(t)\right) w^{\varepsilon, i}\left(t, x_{i}(t)\right) \\
& \leq-\sum_{j=1, \ldots, d} A_{i j} w^{\varepsilon, j}\left(t, x_{j}(t)\right) w^{\varepsilon, i}\left(t, x_{i}(t)\right)
\end{aligned}
$$

where we have used the fact that $\lambda_{, j}^{i} \leq 0$ for $i \neq j$. Applying the comparison principle (see Barles [3, Th 2.4]), we deduce that

$$
m_{i}(t) \leq m_{i}(0)-\int_{0}^{t} \sum_{j=1, \ldots, d} A_{i j} m_{j}(s) m_{i}(s) d s .
$$

Taking the sum over the index $i$, from (4.7) we get that the quantity $m(t)=\sum_{i=1, \ldots, d} m_{i}(t)$ satisfies the following

$$
\begin{aligned}
m(t) & \leq m(0)-\int_{0}^{t} \sum_{i, j=1, \ldots, d} A_{i j} m_{j}(s) m_{i}(s) d s \\
& \leq m(0)=\sum_{i=1, \ldots, d}\left\|\partial_{x} u_{0}^{\varepsilon, i}\right\|_{L^{\infty}(\mathbb{R})} \leq \sum_{i=1, \ldots, d}\left\|\partial_{x} u_{0}^{i}\right\|_{L^{\infty}(\mathbb{R})},
\end{aligned}
$$

where we have used assumption $(K 2)$ and $w^{\varepsilon, i} \geq 0$, for $i=1, \ldots, d$. This proves (1.7).

3- The case where $(K 3)$ holds: We are interested in the following quantity:

$$
m(t)=\max _{i=1, \ldots, d} m_{i}(t)=m_{i_{0}}(t) \quad \text { for some } \quad i_{0}=i_{0}(t) .
$$

We remark that $m \in U S C(0, T)$ and that $m$ is a viscosity subsolution of (4.6), which implies (in the viscosity sense) that

$$
\begin{aligned}
\frac{d}{d t} m(t) & \leq-\sum_{j=1, \ldots, d} \lambda_{, j}^{i_{0}}\left(u^{\varepsilon}\left(t, x_{i_{0}}(t)\right)\right) w^{\varepsilon, j}\left(t, x_{i_{0}}(t)\right) m_{i_{0}}(t) \\
& \leq-\lambda_{, i_{0}}^{i_{0}}\left(u^{\varepsilon}\left(t, x_{i_{0}}(t)\right)\right)\left(m_{i_{0}}(t)\right)^{2}-\sum_{j=1, \ldots, d, j \neq i_{0}} \lambda_{, j}^{i_{0}}\left(u^{\varepsilon}\left(t, x_{i_{0}}(t)\right)\right) w^{\varepsilon, j}\left(t, x_{i_{0}}(t)\right) m_{i_{0}}(t) .
\end{aligned}
$$

By definition of $m_{i_{0}}$, we deduce that 


$$
\begin{aligned}
\frac{d}{d t} m(t) & \leq-\lambda_{, i_{0}}^{i_{0}}\left(u^{\varepsilon}\left(t, x_{i_{0}}(t)\right)\right)\left(m_{i_{0}}(t)\right)^{2}+\sum_{j=1, \ldots, d, j \neq i_{0}}\left[\lambda_{, j}^{i_{0}}\left(u^{\varepsilon}\left(t, x_{i_{0}}(t)\right)\right)\right]^{-} m_{i_{0}}(t) m_{j}(t) \\
& \leq\left(m_{i_{0}}(t)\right)^{2}\left(-\lambda_{, i_{0}}^{i_{0}}\left(u^{\varepsilon}\left(t, x_{i_{0}}(t)\right)\right)+\sum_{j=1, \ldots, d, j \neq i_{0}}\left(\lambda_{, j}^{i_{0}}\left(u^{\varepsilon}\left(t, x_{i_{0}}(t)\right)\right)\right)^{-}\right) \leq 0,
\end{aligned}
$$

where we have used $(K 3)$ and the fact that $w^{\varepsilon, i} \geq 0$. Finally, we integrate in time and obtain that

$$
m(t) \leq m(0)=\max _{i=1, \ldots, d}\left\|\partial_{x} u_{0}^{\varepsilon, i}\right\|_{L^{\infty}(\mathbb{R})} \leq \max _{i=1, \ldots, d}\left\|\partial_{x} u_{0}^{i}\right\|_{L^{\infty}(\mathbb{R})} .
$$

This proves (1.8).

\section{Remark 4.6 (Miscellaneous extensions)}

In Theorem 1.3 we have considered the study of a particular system only to simplify the presentation. This result could be generalized to the following system

$$
\partial_{t} u^{i}+\lambda^{i}(u, x, t) \partial_{x} u^{i}=h^{i}(u, x, t) \quad \text { on } \quad(0,+\infty) \times \mathbb{R} \quad \text { for } \quad i=1, \ldots, d,
$$

with $\lambda^{i}, h^{i} \in W^{1, \infty}\left(\mathbb{R}^{d} \times \mathbb{R} \times(0,+\infty)\right), \partial_{x} h^{i} \geq 0$ and $h_{, j}^{i} \geq 0$ for $i \neq j$ and with moreover one of the following conditions:

$(K 1)^{\prime}\left\{\begin{array}{l}\lambda_{, j}^{i}(u, x, t) \geq 0 \text { and } h_{, j}^{i}(u, x, t) \geq 0 \\ \text { for all }(u, x, t) \in \mathbb{R}^{d} \times \mathbb{R} \times[0,+\infty) \quad \text { and } j \geq i \quad \text { with } i, j \in\{1, \ldots, d\} .\end{array}\right.$

$(K 2)^{\prime}\left\{\begin{array}{l}\lambda_{, j}^{i}(u, x, t) \leq 0 \quad \text { for all } \quad(u, x, t) \in \mathbb{R}^{d} \times \mathbb{R} \times[0,+\infty) \quad \text { and } j \neq i \quad \text { with } i, j \in\{1, \ldots, d\} \\ A_{i j}=\inf _{\substack{u \in \mathbb{R}^{d}, x \in \mathbb{R} \\ t \geq 0}}\left(\lambda_{, j}^{i}(u, x, t)\right) \quad \text { and } \sum_{i, j=1, \ldots, d} A_{i j} \xi_{i} \xi_{j} \geq 0 \quad \text { for } \xi=\left(\xi_{1}, \ldots, \xi_{d}\right) \in[0,+\infty)^{d} .\end{array}\right.$ $(K 3)^{\prime} \quad \lambda_{, i}^{i}(u, x, t) \geq \sum_{i \neq j}\left(\lambda_{, j}^{i}(u, x, t)\right)^{-} \quad$ for all $\quad(u, x, t) \in \mathbb{R}^{d} \times \mathbb{R} \times[0,+\infty) \quad$ and $\quad i=1, \ldots, d$.

\section{Application to the $1 D$ system of isentropic gas dynamics}

In this section we present an application of the results proved previously. More precisely, we study the system of isentropic gas dynamics, defined as follows

$$
\left\{\begin{array}{l}
\partial_{t} \rho+\partial_{x}(\rho u)=0 \\
\partial_{t}(\rho u)+\partial_{x}\left(\rho u^{2}+p(\rho)\right)=0, \quad \text { with } \quad p(\rho)=\frac{(\gamma-1)^{2}}{4 \gamma} \rho^{\gamma} \\
u(0, x)=u_{0} \quad \text { and } \quad \rho(0, x)=\rho_{0} \geq 0
\end{array}\right.
$$


where $\gamma>1$ and respecting the usual notation for the physical quantities: $\rho$ represents the density of the fluid, $u$ is the velocity of the fluid and $p$ the pressure. In what follows, we present an application of Theorem 1.1 and 1.3 (proved in the present paper) on system (5.1).

First of all, we remark that system (5.1) is a diagonalizable hyperbolic system. Indeed, in the case where $\rho>0$ and $(\rho, u)$ is a smooth solution, we can check easily that the following two variables

$$
r_{1}=u+\frac{2 c}{\gamma-1} \quad \text { and } \quad r_{2}=u-\frac{2 c}{\gamma-1}, \quad \text { where } \quad c=\sqrt{\frac{\gamma p}{\rho}}=\frac{(\gamma-1)}{2} \sqrt{\rho^{\gamma-1}},
$$

satisfy the following diagonal system:

$$
\left\{\begin{array}{l}
\partial_{t} r_{1}+\lambda^{1}\left(r_{1}, r_{2}\right) \partial_{x} r_{1}=0 \\
\partial_{t} r_{2}+\lambda^{2}\left(r_{1}, r_{2}\right) \partial_{x} r_{2}=0 \\
\text { with initial data } r_{1}^{0}, r_{2}^{0}
\end{array}\right.
$$

where $\lambda^{1}$ and $\lambda^{2}$ are defined as follows

$$
\left\{\begin{array}{l}
\lambda^{1}\left(r_{1}, r_{2}\right)=\frac{r_{1}+r_{2}}{2}+\frac{\gamma-1}{4}\left(r_{1}-r_{2}\right)=u+c \\
\lambda^{2}\left(r_{1}, r_{2}\right)=\frac{r_{1}+r_{2}}{2}-\frac{\gamma-1}{4}\left(r_{1}-r_{2}\right)=u-c .
\end{array}\right.
$$

Moreover, we have

$$
\left(\lambda_{, j}^{i}\left(r_{1}, r_{2}\right)\right)_{i, j=1,2}=\left(\begin{array}{ll}
\frac{1}{2}+\frac{\gamma-1}{4} & \frac{1}{2}-\frac{\gamma-1}{4} \\
\frac{1}{2}-\frac{\gamma-1}{4} & \frac{1}{2}+\frac{\gamma-1}{4}
\end{array}\right) .
$$

In the case $\gamma>1$, this matrix satisfies the assumptions, $(H 2),(K 3)$ and $(H 2)^{\prime}$, of Theorems 1.1, 1.3 and 2.1. In the following, we show some existence and uniqueness results for system (5.1) applying Theorems 1.1, 1.3 and 2.1.

Firstly, we start with the study of system (5.2) and we consider the following assumptions

$$
\begin{aligned}
& (A 1) \quad r_{1}^{0}, r_{2}^{0} \in L^{\infty}(\mathbb{R}) \text { and } \partial_{x} r_{1}^{0}, \partial_{x} r_{2}^{0} \geq 0 . \\
& (A 2) \quad \partial_{x} r_{1}^{0}, \partial_{x} r_{2}^{0} \in L \log L(\mathbb{R}) . \\
& (A 2)^{\prime} \quad r_{1}^{0}, r_{2}^{0} \in \operatorname{Lip}(\mathbb{R}) .
\end{aligned}
$$

The following existence and uniqueness results for the diagonal system (5.2) hold. 
Theorem 5.1 (Diagonal isentropic gas dynamics system)

Assume (A1) and $\gamma>1$. Then, we have

i) Existence and uniqueness of a continuous solution:

Existence: Under assumption $(A 2)$, system (5.7) has a continuous solution $\left(r_{1}, r_{2}\right)$ on $[0,+\infty) \times$ $\mathbb{R}$ satisfying $(A 1)$ and $(A 2)$ for all $t \geq 0$. Moreover, if $r_{0}^{1}-r_{0}^{2} \geq 0$, then $r^{1}-r^{2} \geq 0$ for all $t \geq 0$.

Uniqueness: Furthermore, if we assume $(A 2)$ and

$$
r_{1}^{0} \geq \Lambda_{1}>\Lambda_{2} \geq r_{2}^{0}
$$

then the previous solution $\left(r_{1}, r_{2}\right)$ is the unique continuous vanishing viscosity solution (in the sense of Definition 3.6).

ii) Existence and uniqueness of $W^{1, \infty}$ solution:

Assume $(A 2)^{\prime}$, then system (5.8) has a unique solution $\left(r_{1}, r_{2}\right) \in\left[W^{1, \infty}([0,+\infty) \times \mathbb{R})\right]^{2}$ satisfying $(A 1)$ and $(A 2)^{\prime}$ for all $t>0$. Moreover, if $r_{0}^{1}-r_{0}^{2} \geq 0$, then $r^{1}-r^{2} \geq 0$ for all $t \geq 0$.

Proof of Theorem 5.1:

Proof of i): We apply Theorem 2.1, which proves that, under the assumptions $(A 1)$ and $(A 2)$, system (5.2) admits a solution $\left(r_{1}, r_{2}\right) \in[C([0,+\infty) \times \mathbb{R})]^{2}$ satisfying $(A 1)$ and $(A 2)$ for all $t \geq 0$.

We now want to prove that, if $r_{1}^{0}-r_{2}^{0} \geq 0$, then $r_{1}-r_{2} \geq 0$ for all $t \geq 0$. To this end, we recall that by Theorem 2.1, we know that $r_{1}=\lim _{\varepsilon \rightarrow 0} r_{1}^{\varepsilon}$ and $r_{2}=\lim _{\varepsilon \rightarrow 0} r_{2}^{\varepsilon}$, where $\left(r_{1}^{\varepsilon}, r_{2}^{\varepsilon}\right)$ is the solution of the following regularized parabolic system

$$
\partial_{t} r_{i}^{\varepsilon}+\lambda^{i}\left(r_{1}^{\varepsilon}, r_{2}^{\varepsilon}\right) \partial_{x} r_{i}^{\varepsilon},=\varepsilon \partial_{x x} r_{i}^{\varepsilon}, \quad \text { for } \quad i=1,2
$$

with regular initial data $r_{1}^{0, \varepsilon}, r_{2}^{0, \varepsilon}$ (see Theorem 2.1). To simplify, we set $r^{\varepsilon}=r_{1}^{\varepsilon}-r_{2}^{\varepsilon}$, using the regularized parabolic system, we can see that $r^{\varepsilon}$ satisfies the following equation

$$
\partial_{t} r^{\varepsilon}=-\left(\frac{r_{1}^{\varepsilon}+r_{2}^{\varepsilon}}{2}\right) \partial_{x} r^{\varepsilon}-\frac{\gamma-1}{4} r^{\varepsilon} \partial_{x}\left(r_{1}^{\varepsilon}+r_{2}^{\varepsilon}\right)+\varepsilon \partial_{x x} r^{\varepsilon} .
$$

Using the maximum principle theorem for parabolic equations (see Lieberman [21, Th 2.10]), we know that the following property holds:

$$
\text { If } r^{\varepsilon}(0, x) \geq 0, \quad \text { then } \quad r^{\varepsilon}(t, x) \geq 0 \text { for all } t>0 .
$$

We pass to the limit $\varepsilon \rightarrow 0$ and obtain that $r(t, x) \geq 0$. This proves the existence result announced in i). The proof of the uniqueness result is direct application of Theorem 1.1.

Proof of ii): The proof of ii) is similar to the proof of i). Indeed, we apply Theorem 1.3 (with assumption (K3)), which proves that, under the assumptions $(A 1)$ and $(A 2)^{\prime}$, system (5.2) admits a solution $\left(r_{1}, r_{2}\right) \in\left[W^{1, \infty}([0,+\infty) \times \mathbb{R})\right]^{2}$ satisfying $(A 1)$ and $(A 2)^{\prime}$ for all $t \geq 0$. 
Moreover, we can prove as in the proof of i) that if $r_{1}^{0}-r_{2}^{0} \geq 0$, then $r_{1}-r_{2} \geq 0$ for all $t \geq 0$. Let us mention that in the case of Lipschitz solutions, we can also prove the following result: if $r_{1}^{0}-r_{2}^{0} \geq \Lambda>0$, then $r_{1}-r_{2} \geq \Lambda e^{-\alpha t}>0$ for all $t \geq 0$, with $\alpha=\frac{\gamma-1}{2} \max _{i=1,2}\left\|\partial_{x} r_{i}^{0}\right\|_{L^{\infty}(\mathbb{R})}$.

Before going into the proof of Theorem 1.4, we need the following technical lemma.

\section{Lemma 5.2 (From Diagonal system to nondiagonal)}

Let us consider two functions $r_{1}, r_{2} \in C([0,+\infty) \times \mathbb{R}) \cap W_{\text {loc }}^{1,1}([0,+\infty) \times \mathbb{R})$ satisfying $r_{1}-r_{2} \geq 0$ on $[0,+\infty) \times \mathbb{R}$, with $\left(r_{1}, r_{2}\right)$ solution of (5.8). Then the following functions

$$
u=\frac{r_{1}+r_{2}}{2} \quad \text { and } \quad \rho^{\theta}=\frac{r_{1}-r_{2}}{2} \text { where } \theta=\frac{\gamma-1}{2}
$$

solve the following system

$$
\left\{\begin{array}{l}
\partial_{t}\left(\rho^{\theta}\right)+u \partial_{x}\left(\rho^{\theta}\right)+\theta \rho^{\theta} \partial_{x} u=0 \\
\partial_{t} u+u \partial_{x} u+\theta \rho^{\theta} \partial_{x}\left(\rho^{\theta}\right)=0 .
\end{array}\right.
$$

Reciprocally, if $\rho^{\theta}, u \in C([0,+\infty) \times \mathbb{R}) \cap W_{\text {loc }}^{1,1}([0,+\infty) \times \mathbb{R})$ (with $\rho^{\theta} \geq 0$ ) is solution of (5.5) then $r_{1}, r_{2}$ defined in (5.4), solve (5.9).

With a simple computation we can check the result (see also Serre [26, Vol II]).

\section{Proof of Theorem 1.4:}

Firstly we prove the existence and uniqueness of a Lipschitz solution announced in Theorem 1.4 ii).

Proof of ii): We prove the result three steps.

Step 1. (Existence and uniqueness of $\left.\left(r_{1}, r_{2}\right)\right)$ : We remark that, if $u_{0}$ and $\rho_{0}^{\theta}$ satisfy assumptions $(J 1)$ and $(J 2)^{\prime}$, then the functions $r_{1}^{0}=u_{0}+\rho_{0}^{\theta}$ and $r_{2}^{0}=u_{0}-\rho_{0}^{\theta}$, where $\theta=\frac{\gamma-1}{2}$, satisfy assumptions $(A 1)$ and $(A 2)^{\prime}$. Now, we consider system $(5.2)$ with the following initial data $r_{1}^{0}=u_{0}+\rho_{0}^{\theta}$ and $r_{2}^{0}=u_{0}-\rho_{0}^{\theta}$. We apply Theorem 5.1 ii), which proves that system (5.2) admits a unique solution $\left(r_{1}, r_{2}\right)$ in $W^{1, \infty}([0,+\infty) \times \mathbb{R})$.

Using the condition $r_{1}^{0}-r_{2}^{0}=2 \rho_{0}^{\theta} \geq 0$, we can also prove, by Theorem 5.1 ii), that $r_{1}-r_{2} \geq 0$ for all $t>0$.

Step 2. (From $\left(r_{1}, r_{2}\right)$ toward $\left.(\rho, u)\right)$ : By Lemma 5.2, it is equivalent to say that $u=\frac{r_{1}+r_{2}}{2}$ and $\rho^{\theta}=\frac{r_{1}-r_{2}}{2} \geq 0$ are in $W^{1, \infty}([0,+\infty) \times \mathbb{R})$ and solution of system (5.5).

We can also see that in the case $1<\gamma \leq 3$ the functions $u$ and $\rho$ defined above belong to $W^{1, \infty}([0,+\infty) \times \mathbb{R})$, and moreover solve the following system

$$
\left\{\begin{array}{l}
\rho^{\theta-1}\left[\partial_{t} \rho+u \partial_{x} \rho+\rho \partial_{x} u\right]=0 \\
\rho \partial_{t} u+\rho u \partial_{x} u+\theta^{2} \rho^{2 \theta} \partial_{x} \rho=0
\end{array}\right.
$$


Using the following result:

$$
\text { If } f \in W^{1, p} \text { for some } p \in[1,+\infty] \text {, then } D f=0 \text { a.e. on the set }\{f=0\} \text {, }
$$

we can rewrite (5.6) as follows

$$
\left\{\begin{array}{l}
\partial_{t} \rho+\partial_{x}(u \rho)=0, \\
\rho \partial_{t} u+\rho u \partial_{x} u+\partial_{x}(p(\rho))=0 .
\end{array}\right.
$$

This shows that $(\rho, u)$ is a solution of system (5.1).

Step 3. (Uniqueness of $(\rho, u))$ : Reciprocally, if $(\rho, u) \in\left[W^{1, \infty}([0,+\infty) \times \mathbb{R})\right]^{2}$ solves (5.7), with $\rho \geq \Lambda>0$, we want to show that $(\rho, u)$ is unique. From Step 1, it is sufficient to show that $r_{1}=u+\rho^{\theta}$ and $r_{2}=u-\rho^{\theta}$ is solution of (5.2). This is easy to see that this is true by reversing the arguments of Step 2.

Now, we prove the existence and uniqueness of a continuous solution announced in Theorem $1.4 \mathrm{i})$.

Proof of i): We proceed as in the proof of ii). We consider system (5.2) with the following initial data $r_{1}^{0}=u_{0}+\rho_{0}^{\theta}$ and $r_{2}^{0}=u_{0}-\rho_{0}^{\theta}$. We apply Theorem 5.1 i) (Existence), we prove that, under the assumption $(J 1)$ and $(J 2)$, system (5.2) admits a continuous solution $\left(r_{1}, r_{2}\right)$ on $[0,+\infty) \times \mathbb{R}$ satisfying $(A 1)$ and $(A 2)$. Since $r_{1}^{0}-r_{2}^{0}=2 \rho_{0}^{\theta} \geq 0$, we know also that $r_{1}-r_{2}=2 \rho^{\theta} \geq 0$, for all $t>0$.

Moreover, if we assume the condition $r_{1}^{0} \geq \Lambda_{1}>\Lambda_{2} \geq r_{2}^{0}$ then in particular we have that $2 \rho^{\theta} \geq \Lambda_{1}-\Lambda_{2}>0$. This proves that system (5.5) is equivalent to system (5.7). By Lemma 5.2, we deduce that it is equivalent to write that $u=\frac{r_{1}+r_{2}}{2}$ and $\rho^{\theta}=\frac{r_{1}-r_{2}}{2}$ are continuous solution of system (5.5) satisfying ( $J 1)$ and (J2) for all $t \geq 0$. We use Theorem 5.1 i) (Uniqueness), which proves that $(\rho, u)$ is the unique continuous vanishing viscosity solution (in the sense of Definition [3.6).

\section{Acknowledgements}

This work was partially supported by the program " $\mathrm{PPF}$, programme pluri-formations mathmatiques financires et EDP", (2006-2010), Marne-la-Vallée University and Ecole Nationale des Ponts et Chausses.

\section{References}

[1] R. A. Adams, Sobolev spaces, Academic Press [A subsidiary of Harcourt Brace Jovanovich, Publishers], New York-London, 1975. Pure and Applied Mathematics, Vol. 65. 
[2] M. Bardi and I. Capuzzo-Dolcetta, Optimal control and viscosity solutions of Hamilton-Jacobi-Bellman equations, Systems \& Control: Foundations \& Applications, Birkhäuser Boston Inc., Boston, MA, 1997. With appendices by Maurizio Falcone and Pierpaolo Soravia.

[3] G. BARLes, Solutions de viscosité des équations de Hamilton-Jacobi, vol. 17 of Mathématiques \& Applications (Berlin) [Mathematics \& Applications], Springer-Verlag, Paris, 1994.

[4] S. Bianchini And A. Bressan, Vanishing viscosity solutions of nonlinear hyperbolic systems, Ann. of Math. (2), 161 (2005), pp. 223-342.

[5] M. Cannone, A. El Haju, R. Monneau, and F. Ribaud, Global existence for a system of non-linear and non-local transport equations describing the dynamics of dislocation densities, Arch. Ration. Mech. Anal., 196 (2010), pp. 71-96.

[6] M. G. Crandall and P.-L. Lions, Condition d'unicité pour les solutions généralisées des équations de Hamilton-Jacobi du premier ordre, C. R. Acad. Sci. Paris Sér. I Math., 292 (1981), pp. 183-186.

[7] R. J. DiPerna, Convergence of approximate solutions to conservation laws, Arch. Rational Mech. Anal., 82 (1983), pp. 27-70.

[8] R. J. DiPerna, Compensated compactness and general systems of conservation laws, Trans. Amer. Math. Soc., 292 (1985), pp. 383-420.

[9] A. El HAJJ, Well-posedness theory for a nonconservative Burgers-type system arising in dislocation dynamics, SIAM J. Math. Anal., 39 (2007), pp. 965-986.

[10] A. El Haju and N. Forcadel, A convergent scheme for a non-local coupled system modelling dislocations densities dynamics, Math. Comp., 77 (2008), pp. 789-812.

[11] A. El Haju And R. Monneau, Global continuous solutions for diagonal hyperbolic systems with large and monotone data, J. Hyperbolic Differ. Equ., 7 (2010), pp. 139-164.

[12] J. GLimm, Solutions in the large for nonlinear hyperbolic systems of equations, Comm. Pure Appl. Math., 18 (1965), pp. 697-715.

[13] H. IsHII, On uniqueness and existence of viscosity solutions of fully nonlinear second-order elliptic PDEs, Comm. Pure Appl. Math., 42 (1989), pp. 15-45.

[14] — Perron's method for monotone systems of second-order elliptic partial differential equations, Differential Integral Equations, 5 (1992), pp. 1-24.

[15] H. Ishi And S. KoIKe, Viscosity solutions for monotone systems of second-order elliptic PDEs, Comm. Partial Differential Equations, 16 (1991), pp. 1095-1128.

[16] R. JENSEn, The maximum principle for viscosity solutions of fully nonlinear second order partial differential equations, Arch. Rational Mech. Anal., 101 (1988), pp. 1-27. 
[17] P. D. LAx, Hyperbolic systems of conservation laws and the mathematical theory of shock waves, Society for Industrial and Applied Mathematics, Philadelphia, Pa., 1973. Conference Board of the Mathematical Sciences Regional Conference Series in Applied Mathematics, No. 11.

[18] P. LeFloch, Entropy weak solutions to nonlinear hyperbolic systems under nonconservative form, Comm. Partial Differential Equations, 13 (1988), pp. 669-727.

[19] P. LeFloch And T.-P. Liu, Existence theory for nonlinear hyperbolic systems in nonconservative form, Forum Math., 5 (1993), pp. 261-280.

[20] T. T. LI, Global classical solutions for quasilinear hyperbolic systems, vol. 32 of RAM: Research in Applied Mathematics, Masson, Paris, 1994.

[21] G. M. Lieberman, Second order parabolic differential equations, World Scientific Publishing Co. Inc., River Edge, NJ, 1996.

[22] P.-L. Lions, B. Perthame, And P. E. Souganidis, Existence and stability of entropy solutions for the hyperbolic systems of isentropic gas dynamics in Eulerian and Lagrangian coordinates, Comm. Pure Appl. Math., 49 (1996), pp. 599-638.

[23] M. MERCIER, Étude de différents aspects des EDP hyperboliques: persistance d'onde de choc dans la dynamique des fluides compressibles, modélisation du trafic routier, stabilité des lois de conservation scalaires, 2010. Thèse, Université Lyon 1, 2010.

[24] A. PAZY, Semigroups of linear operators and applications to partial differential equations, vol. 44 of Applied Mathematical Sciences, Springer-Verlag, New York, 1983.

[25] F. Poupaud, Global smooth solutions of some quasi-linear hyperbolic systems with large data, Ann. Fac. Sci. Toulouse Math. (6), 8 (1999), pp. 649-659.

[26] D. Serre, Systems of conservation laws. I, II, Cambridge University Press, Cambridge, 1999-2000. Geometric structures, oscillations, and initial-boundary value problems, Translated from the 1996 French original by I. N. Sneddon. 